THIS PAPER HAS BEEN ACCEPTED FOR PUBLICATION AT

SOCIAL PSYCHOLOGICAL AND PERSONALITY SCIENCE 


\title{
Incivility is Rising among American Politicians on Twitter
}

\author{
Jeremy A. Frimer ${ }^{1 *}$, Harinder Aujla ${ }^{1}$, Mathew Feinberg ${ }^{2}$, Linda J. Skitka ${ }^{3}$, \\ Karl Aquino ${ }^{4}$, Johannes C. Eichstaedt ${ }^{5}$ \& Robb Willer ${ }^{6}$
}

${ }^{1}$ University of Winnipeg, Department of Psychology, 515 Portage Avenue, Winnipeg, MB, Canada R3B 2E9

${ }^{2}$ University of Toronto, Rotman School of Management, 105 St. George Street, Toronto ON, Canada, M5S 3E6

${ }^{3}$ University of Illinois at Chicago, Department of Psychology, 1007 W. Harrison St., 1009 BSB, Chicago, IL 60607-7137

${ }^{4}$ University of British Columbia, Sauder School of Business, Vancouver, BC, Canada, V6T 1 Z2

${ }^{5}$ Stanford University, Department of Psychology \& Institute for Human-Centered A.I., 450 Jane Stanford Way, Bldg. 420, Stanford, CA 94305

${ }^{6}$ Stanford University, Department of Sociology, 450 Serra Mall, Bldg. 120, Stanford, CA 94305

*Correspondence to: Jeremy A. Frimer, University of Winnipeg, Department of Psychology, 515

Portage Avenue, Winnipeg, MB, Canada R3B 2E9.j.frimer@uwinnipeg.ca

Acknowledgments: We thank Pablo Barbera, Herbert Chang, Ari Decter-Frain, James

Druckman, Emilio Ferrara, Amit Goldenberg, Justin Grimmer, John S. Hughes III, Austin van 
Loon, and Jamie Pennebaker for helpful comments, and to Garrick Sherman for assistance with the civilian tweet sample.

Funding: This research was funded by a grant from the Social Science and Humanities Research Council (SSHRC) of Canada to Jeremy A. Frimer (grant \#435-2018-1221).

Competing interests: RW gave a speech at Twitter and received an honorarium. However, RW has no current professional relationships with Twitter and does not expect to receive any future compensation. All other authors declare no competing interests.

Data and materials availability: Data and stimuli are available at https://osf.io/thnuk/. All data is available in the main text, the supplementary materials, or the online repository. 


\title{
Incivility is Rising among American Politicians on Twitter
}

\begin{abstract}
We provide the first systematic investigation of trends in the incivility of American politicians on Twitter, a dominant platform for political communication in the U.S. Applying a validated artificial intelligence classifier to all 1.3 million tweets made by members of Congress since 2009, we observe a 23\% increase in incivility over a decade on Twitter. Further analyses suggest that the rise was partly driven by reinforcement learning in which politicians engaged in greater incivility following positive feedback. Uncivil tweets tended to receive more approval and attention, publicly indexed by large quantities of "likes" and "retweets" on the platform. Mediational and longitudinal analyses show that the greater this feedback for uncivil tweets, the more uncivil tweets were thereafter. We conclude by discussing how the structure of social media platforms might facilitate this incivility-reinforcing dynamic between politicians and their followers.
\end{abstract}

Keywords: Incivility, political polarization, Twitter, social media, affective polarization 


\section{Incivility is Rising Among American Politicians on Twitter}

A thriving representative democracy requires that politicians adhere to a set of informal norms and rules of behavior that help temper antagonisms that can arise when people with differing views attempt to coexist and potentially work together (Levitsky \& Ziblatt, 2018). Among the more commonly discussed norms is one proscribing incivility, which we define as impolite language that observers typically find to be rude and/or disrespectful (Mutz, 2005).

Previous research has established that incivility harms individuals, relationships, and democracies. Being the target of, or merely a witness to, incivility has been shown to divert attention from critical issues (Brady \& Crockett, 2019) and impedes creative, open-minded, and effective cognitive processing (Porath \& Erez, 2009; Riskin et al., 2017). Incivility also tends to evoke anger and a desire to retaliate against the norm violator (Nugier, Niedenthal, Brauer, \& Chekroun, 2007; Wang, Silva, 2018). These reactions can impede the cooperation and joint problem-solving needed for a democracy to function (Anderson et al. 2014). Incivility may at times be of strategic value to politicians seeking to communicate moral clarity, signal their loyalty, fundraise, draw attention to issues, or mobilize their supporters to action (Brooks \& Geer, 2007; Mason, 2018). When taken too far, however, political incivility can undermine respect for alternative viewpoints, erode public trust in the political process, and incite other forms of uncivil, undemocratic behavior (e.g., Mutz, 2015; see Lau, Sigelman, \& Rovner, 2007, for a meta-analysis).

In recent years, scholars, journalists, and citizens have expressed concern about an apparent increase in the level of uncivil rhetoric in public discourse and in particular on social media (Haidt \& Hetherington 2012; Iyengar, Sood, \& Lelkes 2012; Mann \& Ornstein, 2012; 
Schaeffer, 2020; Shea \& Fiorina, 2012). Previous work has focused on media (e.g., Sydnor 2019) and citizens (e.g., Su et al. 2018) as purveyors of incivility, largely neglecting an important and influential source: elected officials themselves. One study (Theocharis, Barberá, Fezekas, \& Popa, 2020) examined trends in elite political incivility over a 2-year period on Twitter and failed to detect any overall trend. We expand this investigation by establishing trends in levels of incivility over the span of a decade in 1.3 million tweets from the Twitter feeds of members of the U.S. Congress.

We focus on the language of elected officials on social media because politicians are tasked with striking compromises with their opponents for democracies to function effectively. Incivility on the part of political elites can undermine this fundamental process in representative democracies. Further, politicians shape the tone of discourse in the mass public, influencing how citizens communicate with one another on potentially divisive issues (Huddy \& Yair, 2019). We focus on social media and Twitter in particular as it is among the primary modes by which U.S. politicians communicate with one another and the public (Pew Research Center, 2020; see the Discussion for an exploration of trends in incivility in other forums).

Prior research explains the rise of related forms of polarization with reference to a weakening of social norms prohibiting intergroup aggression (Iyengar \& Westwood, 2014), the emergence of political action committees ("super PACs"; Haidt \& Hetherington, 2012), partisan media (e.g., Fox News, MSNBC) and echo chambers (Rountree, 2019), and to an increase in negative political campaigning (Iyengar, Sood, Lelkes, 2012). We propose a novel explanation. Drawing from critical observations about the structure of social media, coupled with reinforcement learning principles (Skinner, 1948; Thorndike, 1898), we propose that political 
incivility might be rising on social media because of the operative social feedback mechanisms on the platform that incentivize incivility.

Twitter limits and shapes the manner in which people interact. The most prevalent feedback mechanisms are the options to "reply," "retweet" (share) or "like" a message, whereas other reactions, such as "disliking" are not on the menu of possible responses. The platform publicly displays, as metrics, tallies of how many times each of these occurred. Twitter users including politicians might attend to "reply", "retweet", and "like" counts as indicators of the social response to the original message.

The available scientific evidence appears to suggest that incivility is well-received on Twitter. Uncivil and outrageous tweets tend to be rewarded with greater attention in the form of "retweets" (e.g., Brady, Wills, Jost, Tucker, \& Van Bavel, 2017) and we find that uncivil tweets also receive many "likes". Politicians seeking to serve their constituents might interpret these metrics to mean that their followers approve of their messaging. We propose and present evidence that politicians learn from these social rewards and follow with further incivility in subsequent messages, yielding rising incivility on the platform.

\section{The Present Study}

We tested whether incivility is increasing over time in the Twitter messages of members of the U.S. Congress over the last decade and include presidential analyses for more descriptive purposes as social media has been prevalent for only two presidencies (Obama and Trump). Further analyses examine (and rule out) the possibility that an observed rise in incivility is fully attributable to several potential confounds such as uncivil politicians replacing civil ones in Congress, rising negativity or moral outrage, rising polarization, or demographic shifts in Congress. 
Prior research (Brady et al., 2017) found that uncivil and outrageous tweets tend to receive much publicity (i.e. "retweets"). We corroborate this finding and show that uncivil tweets are also rewarded with many "likes". We then investigate whether the outsized reaction to incivility reinforces the original uncivil behavior and thus encourages more of it. Using a mediational framework, we test whether reactivity to uncivil tweets has also increased over time and whether statistically controlling for this rising reactivity reduces or eliminates the conditioned rate of increase of incivility over time. Noting the limitations of correlational inference, we augment this analysis with a longitudinal framework and a Granger causality analysis, testing whether intermittent periods in which uncivil rhetoric happened to receive an especially strong reaction on the platform were followed by a subsequent escalation of incivility within a Twitter feed.

\section{Methods}

\section{Samples}

To test whether incivility is rising in the public statements of U.S. politicians, we acquired large corpora of tweets by members of the U.S. Congress and U.S. Presidents over the span of a decade. Sample size was determined by public availability: our goal was to gather all publicly available materials. Our smallest sample (Presidential debates) had $80 \%$ power to detect effects of $|d|>0.06$. We report all measures, conditions, and data exclusions, and how we determined sample sizes. This manuscript includes all studies attempted on this line of work.

Congressional tweets. Although Twitter launched in 2006, we analyzed tweets beginning in 2009 because there were very few Congressional tweets prior. We therefore acquired the Twitter feeds of all members of the 111th-116th U.S. Congresses (2009-2019) and removed 350,039 "retweets," leaving $N=1,293,753$ original tweets in this sample. The first step 
to obtaining this sample was to gather the Twitter handles of all members of Congress. For the latest (116th) Congress in the sample, a Twitter list (https://twitter.com/cspan/lists/members-ofcongress/members) provided the handles. For previous Congresses, we searched Twitter manually for each member and used their verified account or whichever was most evidently their professional Twitter account. For the 1003 persons that served in the 111th-116th Congresses, we successfully gathered 841 handles (84\%). In the 111th - 116th Congresses, we identified 404, $458,503,513,535$, and 535 handles, respectively. With each tweet's text, we collected the date and the number of "likes" and "retweets" it received.

To acquire the sample, we used a Python program (tweets_sentiment_congress_heads.py; see https://osf.io/thnuk/?view_only=d461532681bc49569d4a8222fe209644) to provide developer credentials via the Tweepy API (http://tweepy.readthedocs.io/en/v3.8.0/streaming_how_to.html) to collect tweet text, timestamps, favorites (i.e., "likes"), and "retweet" counts for each handle (at the time of data collection). We removed duplicate tweets. The Tweepy API and other interfaces for the Twitter API permits collecting up to 3240 tweets per handle which was sufficient to collect a complete set of tweets for most handles. Some handles had more than 3240 tweets, meaning that the data set did not include all of their tweets. Moreover, politicians that were more active on Twitter (operationalized as the number of tweets collected per handle) were also more uncivil (operationalized as the average incivility across all of their tweets), $r(830)=.12, p<.001$. This introduces a potential confound when observing changes in incivility over time. We address this confound by including models that examine whether incivility increased over time within Twitter feeds.

In this (Congressional) and the Presidential Twitter samples, we excluded "retweets" for 
three reasons. First, "retweets" do not contain content written by the handle owner or his/her staff. Second, "retweeting" can sometimes but does not always signify an endorsement (see Study S4). And third, Twitter assigns "likes" of "retweets" to the original tweet, not to the "retweet" itself. All "retweets"" "like" counts are identically zero and are therefore not amenable to testing our metric reinforcement explanation for the proposed rise in online incivility. We did not include analyses of "retweets with comments" because this feature was rare in this data set and unavailable before 2015.

Both the "likes" and "retweets" metrics had skewed distributions (see Table S1). To correct the skew, we applied log-transformations (after replacing 0s with the score 0.1, following Frimer, 2019) to retain all data and the ordinal nature.

Presidential tweets. For descriptive purposes, we collected all 8,189 original tweets from Barack Obama’s Twitter handle (@barackobama) and 9,503 tweets from Donald Trump’s (@ realdonaldtrump) while each of them was in office. Tweets from Barack Obama were collected using https://github.com/twintproject/twint for 2012-2017 and by manually acquiring tweets issued prior to 2012. Tweets from Donald Trump were downloaded from http://www.trumptwitterarchive.com. Tweets were restricted to the time that each President was in office and concluded on 21 November 2019. With each tweet's text, we collected the date and the number of "likes" and "retweets" it received. Like in the Congressional Twitter sample, both metrics had skewed distributions. We corrected for skew in the same way as for the Congressional tweets.

Civilian tweets. To assess whether changes over time in Congressional incivility might be attributable to a general rise in incivility on Twitter (i.e. among non-politicians too), we collected a pseudo-random sample of 998,935 tweets from the U.S. over the same time period, 
using approximately 100,000 tweets per year.

\section{Measures of Incivility}

Analyzing the level of incivility of each statement in such large corpora is infeasible using human judges. Therefore, we used four text analysis tools to quantify the level of incivility in texts. The primary tool was PerspectiveAPI's "toxicity", which we independently validated (see Study S1) . PerspectiveAPI scores text for the level of incivility (referred to as "toxicity" in the PerspectiveAPI documentation) on a continuous scale from 0 to 100 , reflecting the probability that an observer would find the tweet to be rude or disrespectful. It was designed to assist with moderating discussion forums (Adams, 2018) and trained using machine learning on hundreds of thousands of texts that had been annotated by thousands of human judges. (https://cran.r-project.org/web/packages/peRspective/peRspective.pdf). We independently validated the accuracy of PerspectiveAPI at judging levels of incivility (convergent validity with human observers $r=.627$ with $r=.647$ for Democratic observers and $r=.581$ for Republican observers; Study S1). Table 1 presents examples of statements rated as low and high in incivility by the classifier in 2009 and 2019.

Table 1. Illustrative examples of how the AI classifier characterized the levels of incivility in tweets and speeches by members of the U.S. Congress in 2009 and 2019. The AI classifier (Google's Perspective API) scores texts on a scale from 0 to 100, reflecting the probability (0\% to $100 \%$ ) that a human observer would perceive the text to be rude or disrespectful. AI classifier scores converged with those from human ratings of perceived incivility, $r=.628$, and the accuracy was not conditioned by the political leaning of the politician and/or the judge (see Study S1).

\begin{tabular}{|c|c|c|c|}
\hline Year & Mode & High Incivility & Low Incivility \\
\hline & Tweet & $\begin{array}{l}\text { @JoeSestak goes AWOL: Worst attendance out of all } \\
\text { PA Reps, and 13th worst in all of Congress. } \\
\text { Sen. Arlen Specter }(D-P A) \\
\text { AI-scored Incivility }=45.1\end{array}$ & $\begin{array}{l}\text { The Stupak and Nelson amendments would disrupt the } \\
30 \text {-yr status quo on abortion coverage to the } \\
\text { disadvantage of womens' reproductive health. } \\
\text { Sen. Arlen Specter }(D-P A) \\
\text { AI-scored Incivility }=6.8\end{array}$ \\
\hline 2009 & Speech & $\begin{array}{l}\text { The Pelosi health mandate bill ... is going to destroy } \\
\text { our economy... This is a dead, rotten, stinking fish that } \\
\text { the Speaker is trying to force down the throats of the } \\
\text { American people... } \\
\text { Rep. Keith Broun }(R-G A) \\
\text { AI-scored Incivility }=46.0\end{array}$ & $\begin{array}{l}\text { As House Republicans offer plans and ideas to get our } \\
\text { economy moving again, all we get in return is more of } \\
\text { the same, spending and taxing... we need new ideas ... } \\
\text { to deliver different results. } \\
\text { Rep. Adrian Smith }(R-N E) \\
\text { AI-scored Incivility }=6.5\end{array}$ \\
\hline 2019 & Tweet & $\begin{array}{l}\text { Gov. Northam in Virginia is endorsing infanticide \& } \\
\text { proudly doing it! If that doesn't scare you, it should. It's } \\
\text { horrifying.... } \\
\text { Rep. Kevin Hern (R-OK) }\end{array}$ & $\begin{array}{l}\text {...the total price tag of \#GreenNewDeal would be ... } \\
\text { roughly four times the value of all Fortune } 500 \\
\text { companies combined. That's no deal. } \\
\text { Sen. John Barrasso }(R-W Y)\end{array}$ \\
\hline
\end{tabular}




\begin{tabular}{lll}
\hline & AI-scored incivility $=47.6$ & AI-scored Incivility $=6.4$ \\
\hline Speech & This President and his Cabinet are so out of touch, it is & I have heard stories of patients that are forced to \\
& pathetic... Shame on him and shame on Majority & choose between putting food on the table or paying for \\
& Leader McConnell for refusing to end this shutdown. & lifesaving drugs. Meanwhile, Big Pharma continues to \\
& He, along with the President, need to grow up. & collect hundreds of billions of dollars in profits each \\
& Rep. Jim McGovern $(D-M D)$ & year. This cannot continue. \\
& AI-scored incivility $=48.6$ & Rep. Debbie Mucarsel-Powell $(D-F L)$ \\
& AI-scored incivility $=6.7$
\end{tabular}

To code the incivility of each statement, we had Python code transmit one tweet/speech/debate statement per second to http://perspectiveapi.com. PerspectiveAPI can analyze any text up to 3000 characters in length. PerspectiveAPI toxicity scores were normally distributed (skews < 2), with sample $M \mathrm{~s} \sim 13$ and $S D \mathrm{~s} \sim 10$ (see the Supplement for details). To make results interpretable in real-world terms, incivility scores were retained in their raw form in the main analyses. In effect size analyses, incivility scores were standardized (z-scores).

\section{Covariates}

Any changes over time in the level of incivility in Congressional tweets are correlational and thus prone to third variable explanations. To establish whether changes in incivility over time are attributable to related or more general changes in language, such as rising negativity, we included measures of overall emotional tone and moral outrage. To assess whether the changes are attributable to a shifting political landscape, we included measures of politician partisanship and district safety. And we included demographic variables to assess whether changes in incivility are attributable to shifting demographics (see the Supplement for details about these variables).

\section{Results}

The 11-year analysis of 1.3 million tweets by members of the U.S. Congress revealed that levels of incivility rose on Twitter (Figure 1A). The test was a series of multilevel models, with time in years (mean centered at the year 2016.71) predicting incivility (PerspectiveAPI; 0-100 
scale). In model 1, we included no random effects. This model thus described the total observed change in incivility over time. In this model (see Table 2), incivility increased over time, representing a $23 \%$ rise from 11.8 in 2009 to 14.5 in 2019 . Effect sizes ( $\beta$ s) were computed by standardizing the predictor and outcome variables and re-running the models. This total effect is likely multiply determined, attributable to (a) politicians becoming more uncivil over time, (b) uncivil persons replacing less uncivil persons as members of Congress, and/or (c) particular features of the data collection method that included only more recent tweets from politicians.

Table 2. Changes in levels of incivility among American politicians over time on Twitter. The four multilevel models make different assumptions about the structure of the data and include different controls, but all point to the same conclusion that incivility increased over time.

\begin{tabular}{|c|c|c|c|c|c|}
\hline \multirow[b]{2}{*}{ Model } & \multirow[b]{2}{*}{ Random Effects } & \multicolumn{4}{|c|}{ Increase in Incivility over Time } \\
\hline & & B & $95 \% \mathrm{CI}$ & $\beta$ & $p$ \\
\hline 1. Tweets by politicians & (none) & 0.332 & $0.325,0.340$ & .076 & $<.001$ \\
\hline 2. Tweets by politicians & Intercepts for each politician & 0.231 & $0.219,0.242$ & .053 & $<.001$ \\
\hline $\begin{array}{l}\text { 3. Tweets by politicians; politicians within } \\
\text { sessions of Congress }\end{array}$ & $\begin{array}{l}\text { Intercepts for each politician } \\
\text { and for each session }\end{array}$ & 0.252 & $0.231,0.274$ & .058 & $<.001$ \\
\hline $\begin{array}{l}\text { 4. Tweets by politicians; politicians within } \\
\text { sessions of Congress }\end{array}$ & $\begin{array}{l}\text { Slopes and Intercepts for each } \\
\text { politician and session }\end{array}$ & 0.253 & $0.233,0.275$ & .056 & $<.001$ \\
\hline
\end{tabular}

Model 2 added random intercepts for each politician, effectively controlling for mean level differences between politicians. The observed increase of incivility over time in Model 2 is thus more likely attributable to politicians becoming more uncivil over time. Model 3 added random intercepts for each politician and each session of Congress in a crossed random effects model, thereby controlling for the mean incivility of each cohort and modeling the average increase in incivility within each session, meaning over the two-year period. Finally, Model 4 added random slopes for each politician and for each session, better isolating the change in incivility within each politician's Twitter feed. The effect of incivility over time decreased in magnitude slightly in the latter models but remained significant in all of them. Therefore, selection effects, whereby civil politicians drift away from Twitter and from Congress and 
uncivil politicians gravitate toward the platform and the chamber, might occur but cannot fully account for the observed increase. Politicians seem to have become more uncivil over time.

Incivility also rose in the general population over time, from 21.1 in 2009 to 27.0 in 2019 , representing a $28 \%$ rise (recall that incivility rose by $23 \%$ among politicians over the same time span). An OLS regression confirmed that incivility rose among civilians, $\mathrm{B}=0.167,95 \% \mathrm{CI}=$ $[0.152,0.183], \beta=.022, p<.001$ (see Figure 1A). Interestingly, the rise in Congressional incivility was not explained by the general rise in the civilian population: A multilevel model (Model 2 in Table 2) with time predicting Congressional incivility while controlling for the average civilian incivility in the year of each tweet found that incivility still independently rose among politicians over time, $\mathrm{B}=0.220,95 \% \mathrm{CI}=[0.208,0.232], \beta=.051, p<.001$.

The finding of rising Congressional incivility on Twitter was generally robust across three other linguistic tools that capture elements of uncivil language: hate speech, uncivil words, and swear words (see Table S2). The rise in incivility generalized across the Senate and House of Representatives, as well as across the political spectrum (Figure 1B; see the Supplement for details).

Incivility scores in this study can range from 0 to 100 . Scoring in the $10-25$ range, the average tweets were not particularly uncivil. Even the Twitter feed of President Trump produced scores that averaged around 20 . This observation challenges the notion that most tweets by American politicians (including by President Trump) were uncivil. However, as we will later show, uncivil tweets receive much more attention than civil ones. The population might have had greater exposure to the relatively rare uncivil tweets seem more common than they actually were.

Figure 1. Changes in levels of incivility among American politicians over time on Twitter. Political incivility increased over time on Twitter both in the presidency and among members of the U.S. Congress (A), with the rise being the most pronounced among liberal Democrats (B). This partisan/ideological difference was mostly explained by reactions to President Trump (see the Supplement). Mean level estimates are from separate multilevel models for each year, with random intercepts for each Twitter user. Partisan levels (liberal, moderate, 
conservative) were derived from rollcall voting behavior. Trendlines were inferred from a party $\times$ partisanship $\times$ time multilevel model Error bars represent $95 \%$ CIs.
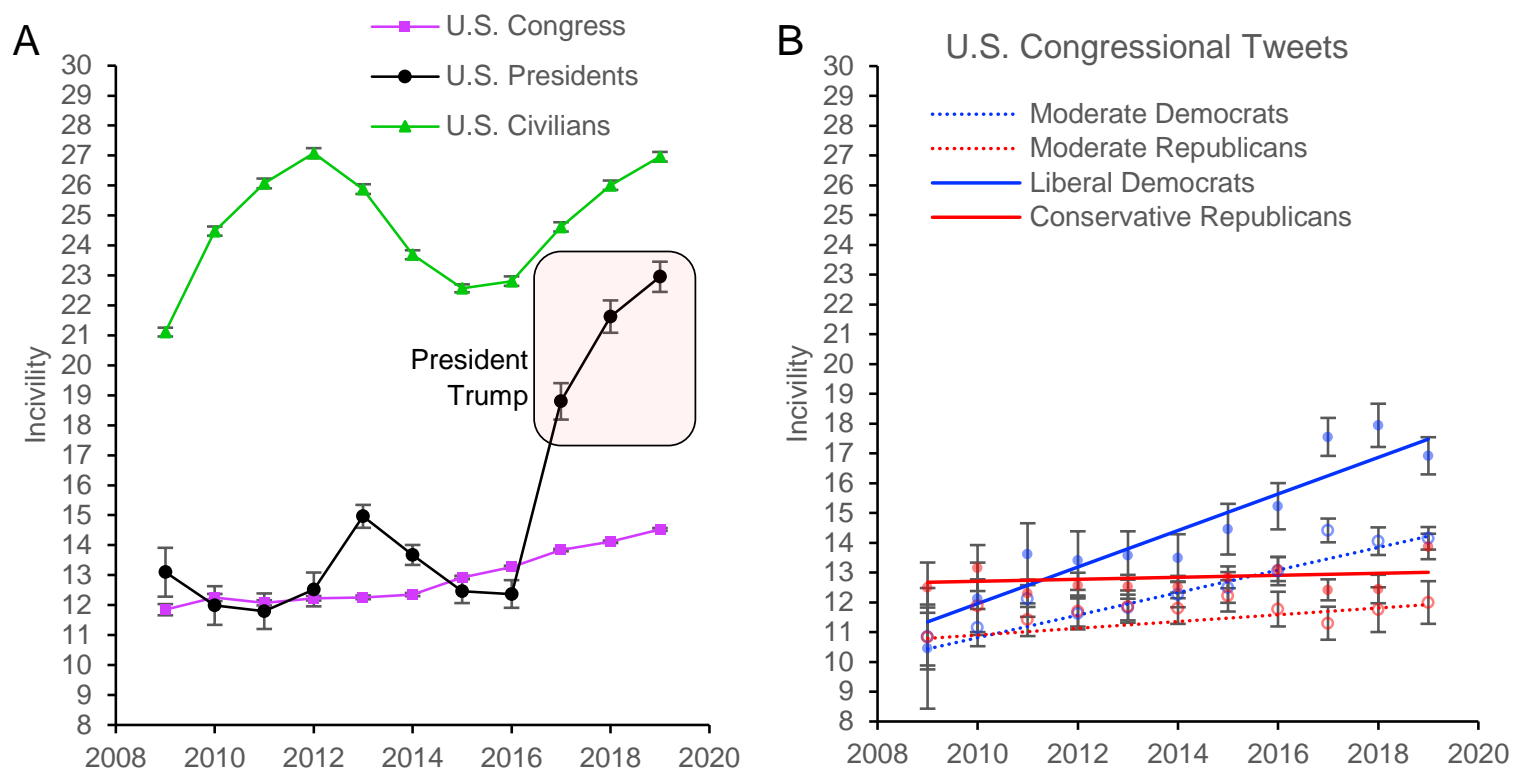

The rise in incivility over time held up when controlling for various potential confounds, each of which may have shifted over time and could have explained the rise. Our analytical approach was to estimate the rate of increase of incivility over time (in years) in a multilevel model (random intercepts for each politician) to establish an unconditioned baseline effect (Figure 2). We then added a covariate, one at a time and with no interaction terms, into a second multilevel model to test whether the conditioned effect of time on incivility remained. Evidence of mediation would be if the conditioned effect decreased or was eliminated.

The rise in incivility was not easily explained by parallel political, demographic, and rhetorical trends. We find that overall negativity in political tweets decreased over time, $\mathrm{B}=$ $1.65,95 \% \mathrm{CI}=[-1.71,-1.60], p<.001$, while incivility increased, meaning that incivility rose $i n$ spite of a tonal shift. Similarly, controlling for the effect of language communicating moral outrage (Brady et al., 2017) only partially accounted for rising incivility that we observed (Figure 2). A number of other potential confounds also stopped short of fully explaining the apparent 
rise. For example, the political polarization of Congress did not explain the rising incivility.

Partisanship has risen in recent decades (Lewis et al., 2019) and partisans were found to be more uncivil than moderates (Figure 1). Yet controlling for partisanship did not account for the rise in incivility. The increase in incivility also remained when controlling for the diversification (along gender and racial lines) and aging of Congress, as well as political considerations (district safety). That said, rising incivility was partly (but not entirely) attributed to members of Congress addressing or talking about President Trump.

Figure 2. Conditioned rise of incivility while controlling for various potential explanations. The model was time predicting incivility while controlling for each potential mediator in separate analyses. The total effect of time on incivility is shown in the black dot with the colored dots being conditioned (indirect) effects of time on incivility. Statistically controlling for metric reinforcement (elevated "likes" and "retweets" from uncivil tweets) reduced or eliminated the observed rise in incivility over time (operationalized as PerspectiveAPI), meaning the metric reinforcement largely explained the rise in incivility. In contrast independently controlling for the effect of demographic, political, and linguistic covariates did not fully account for the observed rise in incivility over time. The increasing incivility over time is in units of change in incivility over 10 years with error bars being $95 \%$ confidence intervals.

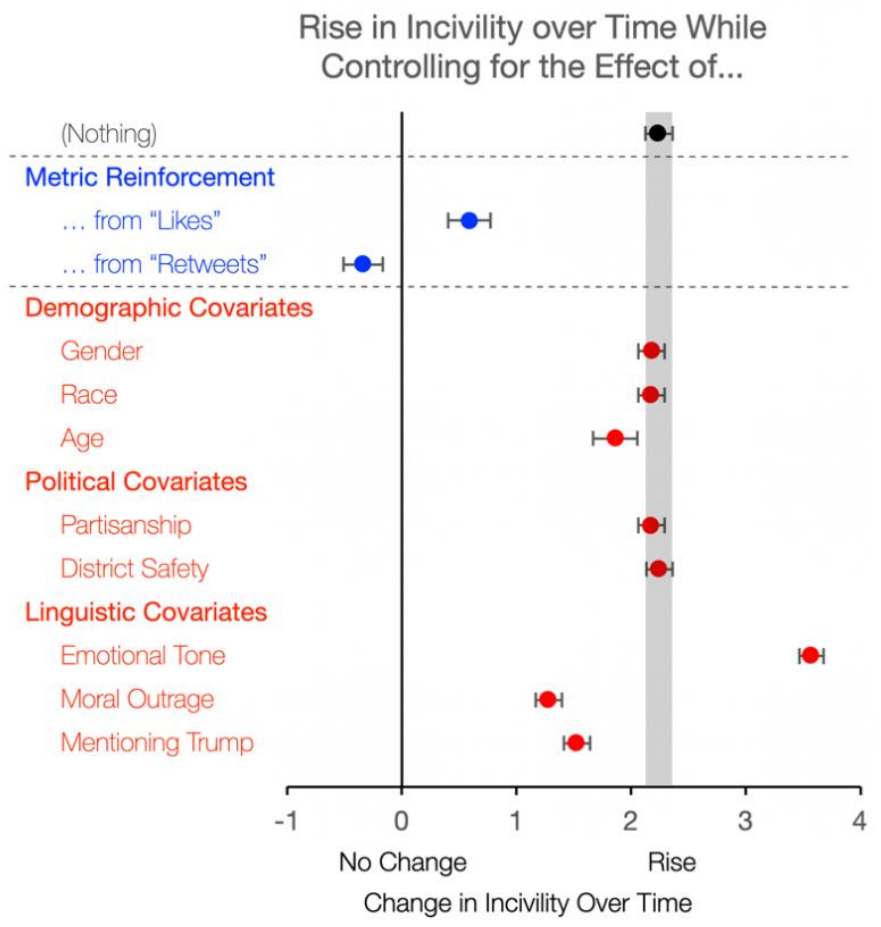

The age of Twitter only spanned the presidencies of Obama and Trump, limiting the scope of an analysis of incivility in the social media feeds of presidents. Incivility levels in President Obama's Twitter feed did not change over time, averaging 13.4 (Figure 1A). When 
President Trump took office, levels of incivility in Presidential tweets jumped to 18.8 in 2017 and kept climbing to 23.0 in 2019, a $76 \%$ increase from 2009 to $2019, d=0.601$. The rise in online incivility during the Trump Presidency means that the rise in incivility in the Twitter feeds of U.S. presidents was not merely attributable to mean level differences between Presidents Obama and Trump.

\section{Reinforcement learning explains the rising incivility}

We found that uncivil tweets were apparently well received on Twitter, and this reception incentivized further incivility. Uncivil tweets received stronger social reactions in the form of many more "retweets" and more "likes" than civil tweets (Figure 3 and the Supplement for analyses). On average, extremely uncivil tweets (incivility $=100$ ) received ten times as many retweets and 8 times as many "likes" as extremely civil tweets (incivility $=0$ ), $p$ s $<.001$. If politicians are trying to be responsive to their constituents, it would be understandable if they were to escalate their incivility when the metrics to which they attend suggest that their incivility was well-received.

Figure 3: Consequences of uncivil tweeting. Uncivil tweets by members of the U.S. Congress received more "retweets" (A) and more "likes" (B) than civil ones. The distributions of "likes" and "retweets" were skewed. We performed log transformations to normalize the data. Analyses were multilevel models, with random intercepts for each politician. We then removed the log transformation to plot on linear scales. Light lines represent 95\% CIs.
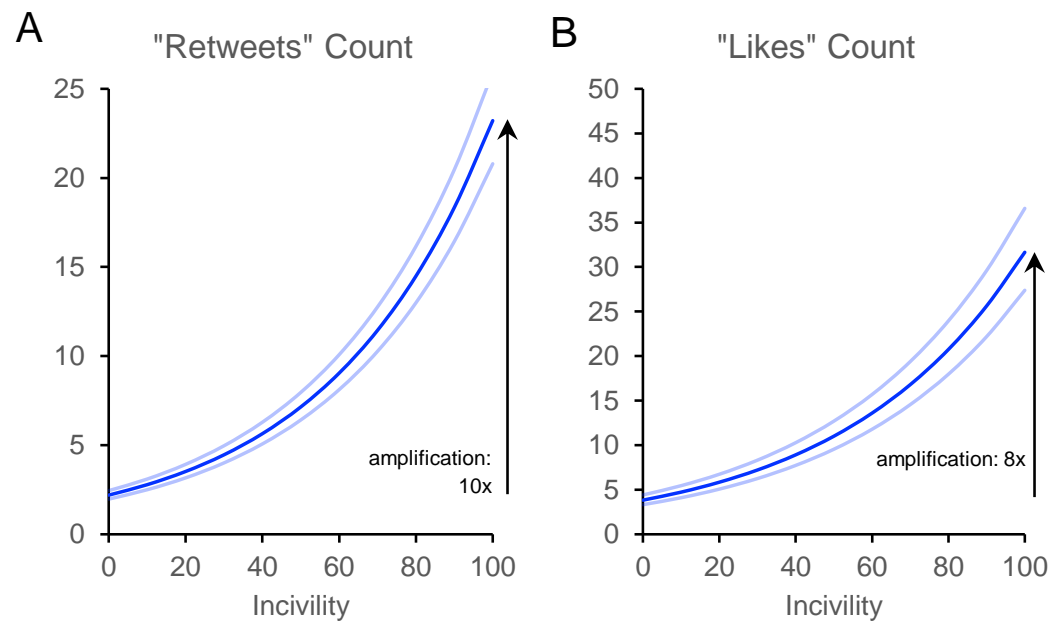
Reactions to uncivil tweets compared to reactions to civil tweets became larger over time (Figure 4). In 2009, uncivil tweets received approximately twice as many "retweets" as civil tweets, but by 2019, uncivil tweets received nearly 15 times as many. Similarly, uncivil tweets received 1.3 times as many "likes" as civil tweets in 2009 but nearly 8 times by 2019 .

We tested whether these increasingly strong reactions to uncivil tweets accounted for the rise in incivility over time on Twitter using a mediational framework and found that they did (Figure 2). A conditioned effect of rising incivility over time was reduced by $74 \%$ when statistically controlling for the increasing reactivity in terms of "likes" and fully eliminated when controlling for reactivity from "retweets". This finding stands in contrast to the limited mediational effects of controlling for various linguistic, political, and demographic factors.

Figure 4. Rewards for uncivil tweeting grew stronger over time. The vertical axis representing the (model-implied) number of times more "retweets" and "likes" uncivil tweets (incivility = 100) received compared to civil tweets (incivility = 0). Error bars are 95\% CIs.

\section{Rewards for Uncivil Tweets}

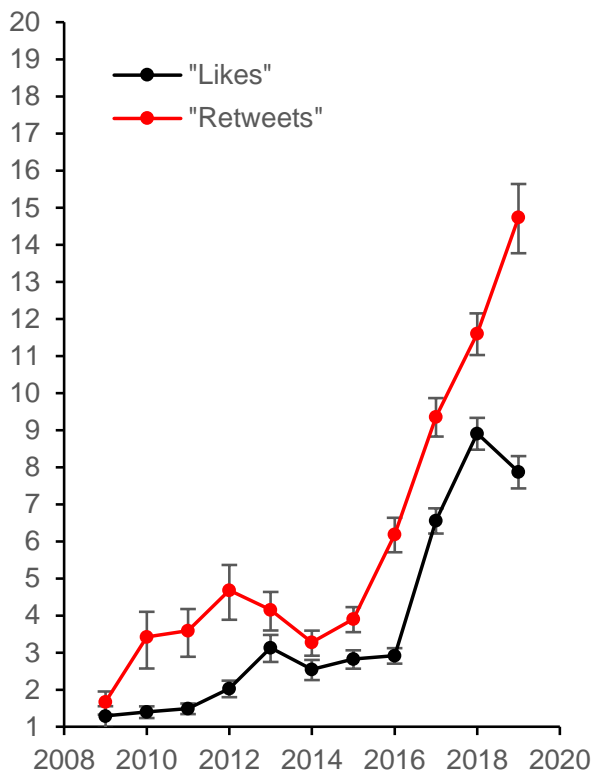

This mediational analysis is correlational and thus prone to reverse causal and third variable interpretations. To more directly test whether reactions to incivility prompted more 
incivility from the politician, we leveraged the longitudinal nature of the data. Time can help tease apart correlational effects into their causal events because events that happen first can cause events that happen second but not vice versa. We therefore examined the reactions that uncivil tweets received were followed by a subsequent uptick in incivility on the part of the politician.

Uncivil tweets tended to receive more "likes" and "retweets" than civil ones (Figure 3). However, there existed variability within each Twitter feed in the degree to which uncivil tweets provoked reactions. There were times for each member of Congress when their civil and uncivil tweets received similar reactions and other times when their incivility received a massive reaction compared to their civil tweets (see Figure S1). We exploited this variability within each Twitter account to test whether times when incivility was particularly well received prompted a larger uptick in incivility than times when incivility was less well received (see Figure S2 for a conceptual illustration).

We formalized this notion with a Granger causality analysis, which allows for a form of causal inference ("Granger causality") from longitudinal data. Formally, a Granger causal effect is established when there is an association between variable $\mathrm{A}$ at time 1 and variable $\mathrm{B}$ at time 2 while controlling for any autocorrelation in variable B at time 1 . In this case, variable $\mathrm{A}$ is the slope between the number of "likes" and the level of incivility in the 50 most recent tweets received. And variable B is levels of incivility in the most recent 50 tweets or the future 50 tweets (equation 1; see the Supplement for a justification of a 50-tweet window and for similar results with 30- and 80-tweet windows; we did not impose date cut-offs):

$$
\text { Incivility }_{\text {future }}=a \cdot \text { Incivility }_{\text {past }}+b \cdot\left(\frac{\# " \text { Likes" }}{\text { Incivility }}\right)_{\text {past }}+\varepsilon
$$

Incivility $_{\text {future }}$ is the average level of incivility of the 50 next original tweets from the handle. Incivility $_{\text {past }}$ is the average level of incivility of the last 50 original tweets from the handle and 
serves as an autocorrelation control variable. And $\left(\frac{\# \text { "Likes" }}{\text { Incivility }}\right)_{\text {past }}$ is the slope of the number of "likes" and the incivility scores of the last 50 tweets from a handle and thus operationalizes reactivity to recent uncivil messages. An analogous formula was applied to reactions in the form of "retweets". The parameter $b$ is an indicator of whether learning has occurred and in what direction. If $b$ is positive, the metric had a reinforcing effect on incivility. We fit these models for each of "likes" and "retweets" to assess which of the two metrics are implicated in learning.

The Granger causality analyses confirmed that politicians reacted to variability in reactions to incivility as theorized, $b_{\text {retweets }}=4.34,95 \%$ confidence interval $=[4.05,4.63], b_{\text {likes }}=$ $3.51,95 \%$ confidence interval $=[3.20,3.82], p s<.001($ see the Supplemental Materials for full details). Together, these results provide converging evidence that politicians learned from their positive experiences with uncivil tweeting and thus doubled down.

\section{Discussion}

We present the first robust evidence that incivility is rising among American politicians on Twitter, a rise that was not explained by a general rise in incivility in the civilian population. The rise appears to be attributable to more uncivil politicians replacing less uncivil politicians in Congress as well as to politicians themselves becoming more uncivil over time. Although the repercussions of this rise in incivility remains to be fully understood, the animosity and distrust it likely breeds are likely having some negative effects on American democracy and governance.

What is proximally causing the rising incivility? We offer the novel explanation that the rise is partly attributable to reinforcement learning on the platform. Uncivil tweets tended to receive many more "retweets" and "likes" than civil tweets. If politicians pay attention to these metrics and desire the attention and approval that they imply, they might learn that uncivil tweeting will help them politically. Both mediational and longitudinal analyses supplied 
supportive evidence of this explanation. The degree to which uncivil tweets received more "retweets" and "likes" than civil ones increased over the years under study; this historical shift mediated the rise in incivility over time. And using a longitudinal framework, we found that the Twitter community's positive reactions to incivility predicted a subsequent uptick in incivility within the Twitter feeds of politicians.

If rising incivility on Twitter is partly due to platform-specific dynamics, as we find they might be, then we might expect to find that incivility has not risen in other contexts where social media dynamics are not in operation. Study S5 examined whether incivility rose in two offline contexts: Congressional speeches during floor debates between 1995-2020 and in Presidential debates between 1976-2016. We found that incivility did not rise and may have even been in decline in these in-person settings. These data lend further credence to the notion that social media might uniquely foster incivility.

This juxtaposition between rising incivility on Twitter and flat or falling incivility in inperson exchanges raises questions about what, specifically, is the critical difference between these contexts that elicit different trends. One possibility is being and feeling physically distant from others on social media has a disinhibiting effect. Put simply, politicians might feel less inclined to be uncivil to a person's face then they do in a private setting with their smart phone.

Another possibility is that features of the social media platform are playing an active role in fomenting incivility. By displaying quantities of reactions (e.g., "likes" counts), social media meaningfully altered the modes by which people provide social feedback to others. Jack Dorsey, the CEO of Twitter, acknowledged that the ramifications of these decisions have yet to be fully understood $^{1}$. We propose that these metrics might not mean what they appear to mean, and that

\footnotetext{
${ }^{1}$ In a 2020 interview, Jack Dorsey acknowledged that the ramifications of presenting quantities of social reactions might be complex and remained poorly understood, "The disciplines that we were lacking in the company in the
} 
this distortion might be partly responsible for the rising incivility. On the one hand, uncivil tweets tended to receive more "likes" than civil ones (Figure 3), implying social approval for incivility. On the other hand, recent research established that people tend to disapprove of uncivil messages (Frimer \& Skitka, 2018, 2020). If observers tend to disapprove of uncivil tweets, why do uncivil tweets receive so many "likes"?

Supplemental studies S2 and S3 made the provocative discovery that the "likes" metric diverges with social approval and therefore signals the opposite of the underlying psychological reality. Paradoxically, we found that the more "likes" a tweet received, the less people actually liked it (see Studies S2 and S3). Figure 4 illustrates the paradoxical divergence of Twitter metrics and social evaluations in two tweets by members of Congress.

Figure 4. Examples of the distorting meaning of Twitter metrics. The top tweet by Rep. D. Johnson (R) is relatively civil and received relatively few "likes" or "retweet," implying that it was not well received. Yet it received positive evaluations from an off-platform panel and a high "likes"-per-"retweet" ratio implying approval. In contrast, the bottom tweet by Rep. J. Kennedy III (D) is relatively uncivil, referring to political opponents as "intellectually \& morally bankrupt". It received many "likes" and "retweet" counts, implying a positive reception. Yet it received negative evaluations from an off-platform panel and a low "likes"-per-"retweet" ratio implying disapproval.

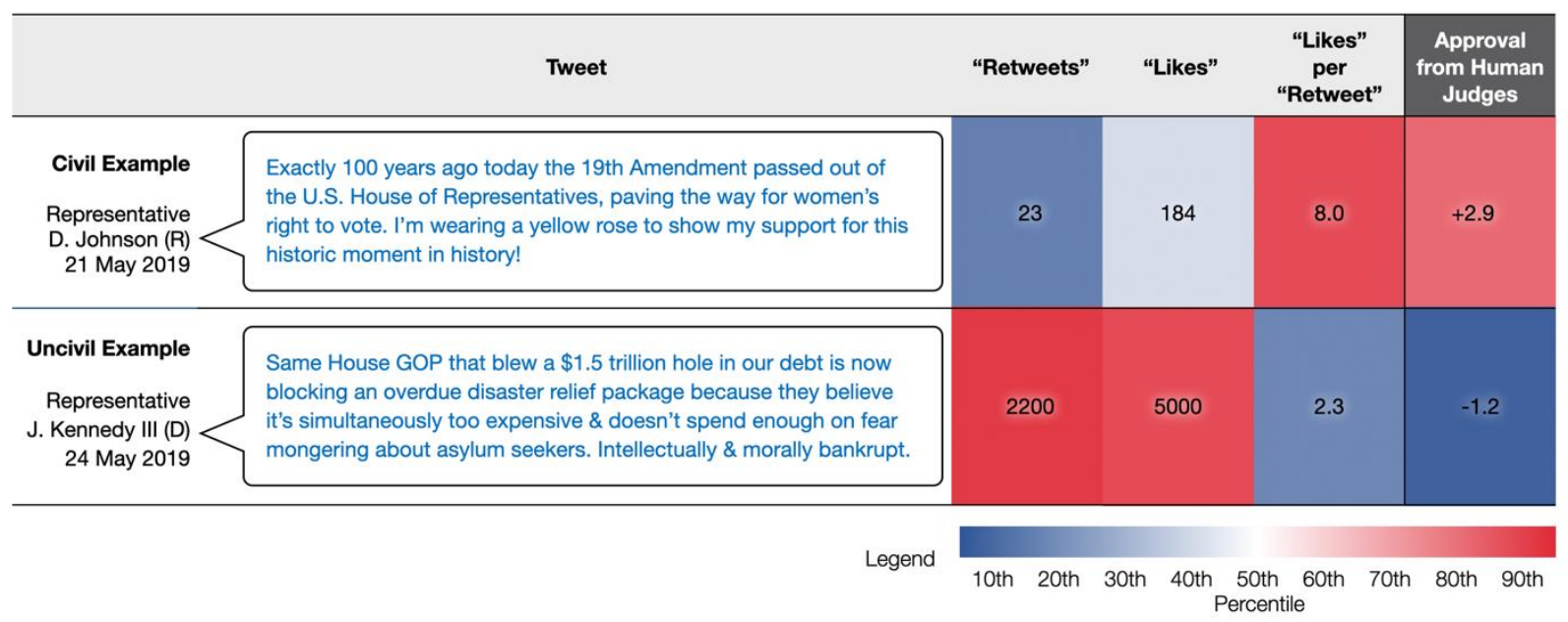

The elevated "likes" counts that uncivil tweets received is likely attributable to their elevated prevalence. Uncivil tweets tend to have a larger audience than civil ones because uncivil

early days that I wish we would have understood and then hired for are like a game theorist to just really understand the ramifications of tiny decisions that we make, such as ... what happens when you put a count next to a "like" button" (Jackson, 2020) 
tweets receive ten times more "retweets" (Figure 3). As the typical Twitter user has 20-400 followers (Wojcik \& Hughes, 2019), each "retweet" creates tens to hundreds of new impressions. Thus, uncivil tweets might be "liked" by a much smaller fraction of those who read them than civil tweets, but the uncivil tweets could still earn a higher absolute number of "likes" if many more people are exposed to those tweets. Although "retweeting" might also appear to signal social approval, Study S4 finds that the "retweeting" of uncivil tweets is driven by their entertainment value rather than by moral approval, a finding consistent with research showing that people feel compelled to pass on information about antisocial behavior without necessarily condoning it (Berger, 2011; Davis \& McLeod, 2003; Feinberg, Willer, Stellar, \& Keltner, 2012). Together, these findings suggest that in spite of appearances the elevated "likes" and "retweets" the uncivil tweets tend to receive do not necessarily imply social approval.

To potentially correct for the confounding effect of exposure on the absolute count of "likes", we created a "likes"-per-"retweet" ratio for each tweet and validated it as a valid indicator of social evaluation (Studies S2 \& S3). Critically, uncivil tweets by members of Congress tended to have a lower "likes"-per-“retweet" ratio than civil tweets (Figures 4 \& 5), implying that the Twitter community, including politicians' like-minded followers, tended to disapprove of uncivil tweets. This finding aligns with decades of research finding that political incivility draws disapproval (Lau, et al., 2007), even from dedicated followers (Frimer \& Skitka, 2018), and further suggests that metrics play a non-neutral role in breeding incivility.

Figure 5: Social disapproval of uncivil tweets. Uncivil tweets by members of the U.S. Congress had a lower "likes"-per-"retweet" ratio than civil tweets. This ratio tracks positively with levels of approval (see Studies S2-S3) meaning that the Twitter community in general did not approve of uncivil tweets in spite of appearances to the contrary. Light lines represent $95 \%$ CIs. 


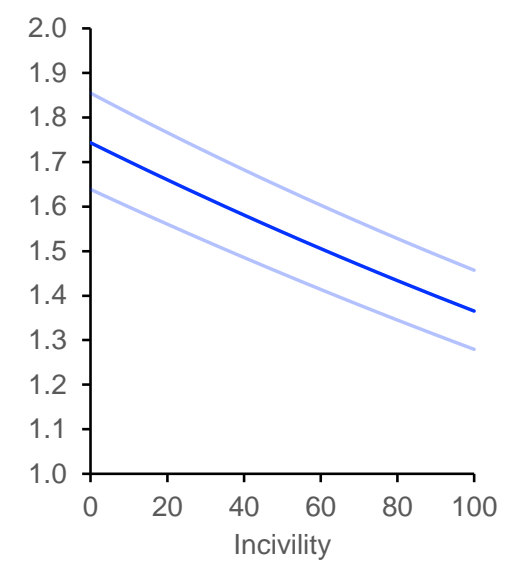

Together, our results suggest that incivility is rising on Twitter in part due to platformspecific features which are amenable to revision. Unless these platform-specific social dynamics are revised, it is likely that the rise in incivility that we observed among American politicians on Twitter will continue to rise. In light of the established deleterious effects that political incivility has on tolerance for alternative viewpoints and public trust in the political process (e.g., Lee et al., 2007; Mutz, 2015), this rise is likely to have negative effects on American democracy and governance.

Data and materials availability: Data and stimuli are available at https://osf.io/thnuk/?view_only=d461532681bc49569d4a8222fe209644. All data is available in the main text, the supplementary materials, or the online repository. 


\section{References}

Adams, C. J. (2018, May 23). New York Times: Using AI to host better conversations. Retrieved from https://www.blog.google/technology/ai/new-york-times-using-ai-host-betterconversations/

Anderson, A.A., Brossard, D., Scheufele, D.A., Xenos, M.A. and Ladwig, P. (2014), The "Nasty Effect:" Online Incivility and Risk Perceptions of Emerging Technologies. Journal of Computer-Mediated Communication, 19: 373-387. doi:10.1111/jcc4.12009

Ansolabehere, S., Iyengar, S., Simon, A., \& Valentino, N. (1994). Does Attack Advertising Demobilize the Electorate? The American Political Science Review, 88(4), 829-838. DOI: $10.2307 / 2082710$

Baumeister, R. F., \& Leary, M. R. (1995). The need to belong: Desire for interpersonal attachments as a fundamental human motivation. Psycho- logical Bulletin, 117, 497-529. http://dx.doi.org/10.1037/0033-2909.117 .3.497

Berger, J. (2011). Arousal increases social transmission of information. Psychological Science, 22(7), 891-893. https://doi.org/10.1177/0956797611413294ååœ

Brady, W.J. \& Crockett, M.J. (2019). How effective is online outrage? Trends in Cognitive Sciences, 23(2), 79-80.

Brady, W. J., Wills, J. A., Jost, J. T., Tucker, J. A., \& Van Bavel, J. J. (2017). Emotion shapes the diffusion of moralized content in social networks. PNAS, 114(28), 7313-7418. Doi: 10.1073/pnas.1618923114

Brady, W. J., Wills, J. A., \& Van Bavel, J. J. (2018, June 13). Brady et al. (2017) PNAS Moral Contagion. Retrieved from osf.io/59uyz 
Brooks, J. B., \& Geer, J. G. (2007). Beyond negativity: The effects of incivility on the electorate. American Journal of Political Science, 51, 1-16. http://dx.doi.org/10.1111/j.15405907.2007.00233.x

Davidson, T., Warmsley, D., Macy, M., \& Weber, I. (2017). Automated hate speech detection and the problem of offensive language, Proceedings of the 11th International AAAI Conference on Web and Social Media, ICWSM '17, Montreal, Canada, 512-51.

Davis, H., \& McLeod, S. L. (2003). Why humans value sensational news: An evolutionary perspective. Evolution and Human Behavior, 24, 208-216. doi:10.1016/S1090$5138(03) 00012-6$

Feinberg, M., Willer, R., Stellar, J. E., \& Keltner, D. (2012). The virtues of gossip: Reputational information sharing as prosocial behavior. Journal of Personality and Social Psychology, 102, 1015- 1030. doi: $10.1037 / \mathrm{a} 0026650$

Fehr, E. \& Gächter,S. (2002). Altruistic punishment in humans. Nature, 415, 137-140.

Ferrara E, Yang Z (2015) Measuring emotional contagion in social media. PLoS ONE 10 (11): e0142390. doi:10.1371/journal.pone.0142390

Frimer, J. A. (2019). Do liberals and conservatives use different moral languages? Two replications and six extensions of Graham, Haidt, and Nosek's (2009) moral text analysis. Journal of Research in Personality. doi: 10.1016/j.jrp.2019.103906

Frimer, J. A. \& Skitka, L. J. (2018). The Montagu Principle. Incivility decreases politicians' public approval, even with their political base. Journal of Personality and Social Psychology, 115(5), 845-866. doi: 10.1037/pspi0000140

Fukuyama, F. (2018). Identity: The demand for dignity and the politics of resentment. Farrar, Strausm and Giroux: New York. 
Haidt, J. \& Hetherington, M. J. (2012, September 2017). Look how far we've come apart. The New York Times. Retrieved from https://campaignstops.blogs.nytimes.com/2012/09/17/look-how-far-weve-come-apart/

Huddy, L. \& Yair, O. (2019). Reducing affective polarization: Warm group relations or policy compromise. Paper presented at the annual meeting of the Western Political Science Association, San Diego.

Hutto, C.J. \& Gilbert, E.E. (2014). VADER: A Parsimonious Rule-based Model for Sentiment Analysis of Social Media Text. Eighth International Conference on Weblogs and Social Media (ICWSM-14). Ann Arbor, MI, June 2014.

Iyengar, S., Sood, G., Lelkes. Y. (2012). Affect, Not Ideology: A Social Identity Perspective on Polarization, Public Opinion Quarterly, 76(3), 405-431, doi: 10.1093/poq/nfs038

Iyengar, S. \& Westwood, S.J. (2015), Fear and loathing across party pines: New evidence on group polarization. American Journal of Political Science, 59, 690-707. doi:10.1111/ajps.12152

Jackson, L. (2020). Jack Dorsey on Twitter's mistakes. Retrieved from https://www.nytimes.com/2020/08/07/podcasts/the-daily/Jack-dorsey-twitter-trump.html

Lau, R. R., Sigelman, L., \& Rovner, I. B. (2007). The effects of negative political campaigns: A meta-analytic reassessment. The Journal of Politics, 69, 1176-1209. Doi: 10.1111/j.14682508.2007.00618.x

Levitsky, S., \& Ziblatt, D. (2018). How democracies die (1st ed.). New York: Crown.

Lewis, J. B., Poole, K., Rosenthal, H., Boche, A., Rudkin, A., \& Sonnet, L. (2019). Voteview: Congressional Roll-Call Votes Database. 
Mann. T. E. \& Ornstein, N. J. (2012). It's even worse than it looks: How the American constitutional system collided with the new politics of extremism. Basic Books: New York.

Mason, L. (2018). Uncivil agreement. University of Chicago Press.

Muddiman, A., McGregor, S. C. \& Stroud, N. J. (2019). (Re)claiming our expertise: Parsing large text corpora with manually validated and organic dictionaries, Political Communication, 36(2), 214-226, Doi: 10.1080/10584609.2018.1517843

Mutz, D. C. (2015). In-your-face politics. The consequences of uncivil media. Princeton $\underline{\text { University Press }}$

Mutz, D. C., \& Reeves, B. (2005). The New Videomalaise: Effects of Televised Incivility on Political Trust. American Political Science Review, 99 (1), 1-15.

Nugier, A., Niedenthal, P. M., Brauer, M., \& Chekroun, P. (2007). Moral and angry emotions provoked by informal social control. Cognition and Emotion, 21, 1699-1720. http://dx.doi.org/10.1080/02699930601124738

Papacharissi, Z. (2004). Democracy online: civility, politeness, and the democratic potential of online political discussion groups. New Media \& Society, 6(2), 259-283. Doi: $10.1177 / 1461444804041444$

Pennebaker, J.W., Booth, R.J., Boyd, R.L., \& Francis, M.E. (2015). Linguistic Inquiry and Word Count: LIWC2015. Austin, TX: Pennebaker Conglomerates (www.LIWC.net).

Pew Research Center (16 July 2020). Congress soars to new heights on social media. Retrieved from https://www.pewresearch.org/internet/2020/07/16/congress-soars-to-new-heightson-social-media/ 
Porath, C. L., \& Erez, A. (2009). Overlooked but not untouched: How incivility reduces onlookers' performance on routine and creative tasks. Organizational Behavior and Human Decision Processes, 109, 29-44. http://dx.doi.org/10.1016/j.obhdp.2009.01.003

Rountree, C. (2019, March 9). How American politics became to uncivil. Retrieved from https://www.al.com/opinion/2016/03/how_american_politics_became_s.html

Riskin, A., Erez, A., Foulk, T. A., Riskin-Geuz, K. S., Ziv, A., Sela, R., . . Bamberger, P. A. (2017). Incivility and medical team performance. Pediatrics, 139, e20162305. http://dx.doi.org/10.1542/peds.2016-2305

Schaeffer, K. (2020). Far more Americans see "very strong" partisan conflicts now than in the last two presidential elections. Retrieved from https://www.pewresearch.org/fact$\underline{\text { tank/2020/03/04/far-more-americans-see-very-strong-partisan-conflicts-now-than-in-the- }}$ last-two-presidential-election-years/

Shea, D. M. \& Fiorina, M. P (2013). Can we talk? The ride of rude, nasty, stubborn politics. Pearson.

Shugars, S., \& Beauchamp, N. (2019). Why keep arguing? Predicting engagement in political conversations online. SAGE Open. Doi: 10.1177/2158244019828850

Skinner, B. F. (1948). Superstition in the pigeon. Journal of Experimental Psychology, 38, 168172.

Snydor, E. (2019). Disrespectful Democracy. Columbia University Press.

Su, L. Y.-F., Xenos, M. A., Rose, K. M., Wirz, C., Scheufele, D. A., \& Brossard, D. (2018). Rude and personal? Comparing patterns of incivility in comments on the Facebook pages of news outlets. New Media \& Society, 20(10), 3678-3699. Doi:

$10.1177 / 1461444818757205$ 
Tausczik, Y. R., \& Pennebaker, J. W. (2010). The psychological meaning of words: LIWC and computerized text analysis methods. Journal of Language and Social Psychology, 29, 24-54.

Theocharis, Y., Barberá, P., Fazekas, Z., \& Popa, S. A. (2020). The dynamics of political incivility on Twitter. SAGE Open. https://doi.org/10.1177/2158244020919447

Thorndike, E. (1898). Some experiments on animal intelligence. Science, 8(18), 818-824

Wang, M. Y. \& Silva, D. E. (2018) A slap or a jab: An experiment on viewing uncivil political discussions on Facebook, Computers in Human Behavior, 81, 73-83, doi: 10.1016/j.chb.2017.11.041.

Wojcik, S. \& Hughes, A. (2019, April 9). Sizing up Twitter Users. Retrieved from https://www.pewresearch.org/internet/2019/04/24/sizing-up-twitter-users/ 


\title{
Incivility is Rising among American Politicians on Twitter
}

\author{
Supplement
}

\section{Overview}

The Supplement presents full analyses the main study, along with methods and results of five supplementary studies. We establish the validity of AI-scored incivility (Study S1). We then examine whether metrics might play an active role in fomenting incivility by distorting negative social reactions to incivility to appear as though the uncivil tweets were well liked (Studies S23). We also show that the elevated "retweeting" of uncivil tweets also has little to do with social approval and is instead linked to their entertaining nature (Study S4). Study S5 then explores trends in incivility in congressional speeches and presidential debates to test whether rising incivility is limited to social media.

\section{Table of Contents}

Congressional Tweets

Study S1: Validity of PerspectiveAPI .

Study S2: Many "Likes” Does Not Imply Approval 1 .......................................................................... 18

Study S3: Many "Likes" Does Not Imply Approval 2 ......................................................................... 20

Study S4: Many "Retweets" Does Not Imply Approval .......................................................................... 25

Study S5: Congressional Speeches \& Presidential Debates ....................................................................... 27

\section{Congressional Tweets}

We tested whether and why incivility is on the rise in the U.S. Congress in a sample of Congressional tweets over an 11-year span. We further examined whether the rise is uniform across the political spectrum, whether a number of potential covariates account for the rise, and whether metric reinforcement explains the rise.

\section{Methods}

Covariates. We operationalized linguistic, political, and demographic covariates in the Congressional Twitter sample to assess whether they explain apparent changes in incivility over time.

Alternative measures of toxicity: swear words. To assess the robustness of trends in incivility over time produced by PerspectiveAPI's toxicity classifier, we used three other previously validated measures of uncivil language. First, we analyzed the density of swear words in each text using Linguistic Inquiry and Word Count's (LIWC; Pennebaker, Booth, Boyd, \& Francis, 2015) built-in swear word dictionary. Word density scores reflect the number of words that match any one of the words in the predefined dictionary, divided by the total number of words in the text. The LIWC swear words dictionary contains 131 swear words and word stems, which include moderately and extremely offensive terms (e.g., geek ${ }^{*}$, wuss, ass, milf*, piss*, fuck, shit*). Swear words were rare (e.g., 99.7\% of Congressional tweets were scored as having 0 swear words). To make results somewhat comparable across measures, we scaled scores so that they could range from 0 to 100 .

Alternative measures of toxicity: Uncivil words. We used the uncivil words dictionary (Muddiman, McGregor \& Stroud, 2019), which is functionally similar to the LIWC swear word 
dictionary however the content of the uncivil words dictionary was curated to capture specifically uncivilwords (e.g., betray*, dishonest*, insan*, sham*, unaccept*) and was validated by Muddiman et al. (2019). Uncivil words were rare (e.g., 96.6\% of Congressional tweets were scored as having 0 uncivil words).

Alternative measures of toxicity: Hate speech. We used a hate speech classifier (Davidson, Warmsley, Macy, \& Weber, 2017), which automatically classifies speech as hate speech (0), offensive speech (1), or neither (2). We scaled scores such that $100=$ hate speech, 50 $=$ offensive speech, and $0=$ neither offensive nor hate speech. Hate speech was rare (e.g., 99.5\% of Congressional tweets were scored as neither offensive nor hate speech).

Emotional tone. Incivility might be confounded with overall negativity. That is, uncivil tweets might be more negative in their emotional tone than civil ones, and negatively valenced tweets tend to receive more "retweets" than positive ones (Ferrara \& Yang, 2015). To address the possibility that any effects that we observe regarding incivility are not explained by more general effects of emotional tone, we scored each text for its emotional tone ("compound" from Vader Sentiment, Hutto \& Gilbert, 2014). Emotional tone scores can range from -1 to 1 and reflect how negative/pessimistic (lower scores) or positive/optimistic (higher scores) the statement is. We scaled the sentiment scores to also range from 0 to 100 .

Moral outrage. A related possibility is that incivility is reducible to the expression of moral outrage whose effects on "retweeting" have already been established (Brady, Wills, Jost, Tucker, \& Van Bavel, 2017). To test whether language expressing moral outrage confound any incivility effects, we analyzed each tweet for the level of moral outrage using the "shared" dictionary from Brady, Wills, \& Van Bavel (2018; words like "fight," "war," "greed," and "punish"; See moral_outrage.counts.py program on https://osf.io/thnuk/?view_only=d461532681bc49569d4a8222fe209644. When analyzing tweets, we used the raw moral outrage counts as the measure of moral outrage (following Brady et al., 2017). We scaled the sentiment scores to also range from 0 to 100.

Partisanship. Highly partisan members of Congress might perceive their role to be to verbally correct and neutralize the other side. We might therefore expect that partisan members of Congress might use more uncivil language than their more moderate colleagues. Previous research found that partisanship has increased over time in U.S. Congress (https://voteview.com/parties/all). Therefore, partisanship might confound any observed rise in incivility over time. To test for this possibility, we operationalized the partisanship of each member of the U.S. Congress with DW-Nominate dimension 1 scores (Lewis et al., 2019). DWNominate relies on multidimensional scaling on the roll call votes in Congress to provide each politicians' ideal point estimates. These points are comparable between branches of government and over time. They range from negative scores (liberal) to scores near 0 (moderate) to positive scores (conservative). We computed partisanship scores as the absolute value of DW-Nominate scores and then computed z-scores so that other variables in our analyses would reflect their effects within the populated range of partisanship.

District safety. In electorally safe districts, members of Congress might feel confident that they will win the general election. Their challenge as politicians is to win primary elections. Uncivilly attacking the other side might signal to the party faithful that the candidate is a loyal defender. Therefore, we might expect that incivility would be higher in safer districts and that reactions to it might be generally more favorable. To test this, we gathered data on all of the results from midterm elections for the House of Representatives from https://graphics.reuters.com/USAELECTION-RESULTS-GRAPHIC/0100813V22J/index.html?exitpoll=true\#section/house, 
https://www.nytimes.com/elections/2014/results/house, and https://www.nytimes.com/elections/2010/results/house/big-board.html and took the share of the popular vote that the winning candidate earned $(M=65.70 \%, S D=12.41 \%)$ as a measure of district safety. We then computed z-scores.

\section{Results}

Metric exhibited skewed distributions (see Table S1) so we applied log transformations to remove the skew prior to analyses.

Table S1. Descriptive statistics of tweets by members of the U.S. Congress sample.

\begin{tabular}{llllll}
\hline \multicolumn{1}{c}{ Metric } & Minimum & Maximum & $M$ & $S D$ & Skew \\
\hline Original Metrics & & & & & \\
$\quad$ Retweets & 0 & 654,152 & 111.140 & $1,370.319$ & 130.401 \\
$\quad$ Likes & 0 & $1,339,670$ & 340.150 & $4,190.749$ & 73.798 \\
$\quad$ "Likes"-per-"Retweet" & 0 & 1440 & 4.678 & 13.809 & 15.584 \\
$\quad$ Incivility & 0.1 & 97.9 & 13.5 & 10.2 & 2.096 \\
Log-Transformed Metrics & & & & & \\
$\quad$ Retweets & -1.000 & 5.816 & 0.568 & 0.970 & 0.361 \\
$\quad$ Likes & -1.000 & 6.127 & 0.781 & 1.148 & 0.212 \\
$\quad$ "Likes"-per-“Retweet" & -4.640 & 3.158 & 0.214 & 0.657 & -0.593 \\
\hline
\end{tabular}

The central finding that incivility, as operationalized by PerspectiveAPI, increased in the Twitter feeds of members of Congress, conceptually replicated in analyses of hate speech, uncivil words, and (marginally with) swear words (which might be explained by the severe floor effect in the latter measure). These findings provide robust evidence that incivility is rising among American politicians on social media.

Table S2. The rise of incivility in U.S. Congress was robust across four linguistic measures of incivility. All predictors were scaled to range from 0 to 100 and time was measured in years. Effects are unstandardized estimates from multilevel models with $95 \%$ confidence intervals in brackets.

\begin{tabular}{lc}
\hline Dependent Variable & $\begin{array}{c}\text { Change over Time } \\
\text { B }[95 \% \text { CI }]\end{array}$ \\
\hline Hate Speech & $0.078[0.071,0.085]^{* * *}$ \\
Swear Words & $5 \times 10^{-5}\left[2 \times 10^{-6}, 1 \times 10^{-4}\right] \dagger$ \\
Uncivil Words & $0.020[0.018,0.022]^{* * *}$ \\
Toxicity & $0.258[0.247,0.270]^{* * *}$ \\
\hline
\end{tabular}

Moderation by political ideology and party. Some experts have suggested that the political right is more uncivil than the political left (Lai, 2018; Mason, 2018). We find that the incivility on Twitter increased across Democratic and Republican, as well as more ideologically moderate and extreme, Congresspeople. However, incivility among Democratic and more liberal 
Congresspeople rose more quickly than among Republican and moderate or conservative Congresspeople. By 2019, Democrats were more uncivil than Republicans.

For this analysis, we included only Democrats and Republicans. We once again used a multilevel model approach, with random intercepts for each Twitter handle. Predictors of incivility were time (recentered at the mean of August 2016, and in units of years), party $(1=$ Republican, $-1=$ Democrat), partisanship (z-scores), and their interactions.

Table S3 shows how we again found a positive effect of time (incivility rose on Twitter), party (Democrats were more uncivil than Republicans), and partisanship (partisans were more uncivil than moderates), along with all higher order interactions. To test whether incivility rose within each party, we computed time $\times$ partisanship full factorial models for each party. For each party, we observed main effects of time (incivility was on the rise on Twitter) and partisanship, as well as interactions. We therefore recentered the partisanship variable at $+/-1 S D$ from the $M$ and reran the time $\times$ partisanship analyses, taking the main effect of time as the unstandardized estimate for each level of partisanship. These analyses revealed that incivility increased among all political groups (liberal and moderate Democrats and conservative and moderate Republicans). However, the greatest rise was found on the political left. In rank order of rate of increase, incivility increased the most among liberal Democrats, followed by moderate Democrats, and then moderate Republicans, and finally conservative Republicans.

Table S3. Results from multilevel models testing whether incivility has increased over time on Twitter and whether the rate of increase has differed between Democrats and Republicans of varying levels of partisanship. Partisanship scores were standardized (z-scores). Time was recentered around the mean and in units of years. Party was effect coded as -1 (Democrats) and 1 (Republicans). Independents were excluded from this analysis. To test for effect of time for moderate and partisan Democrats and Republicans, we recentered partisanship at +/ 1 SD from the M, re-ran the Time $\times$ Partisanship model and took the effect of time as the simple slope for that particular political demographic. Numbers in brackets represent $95 \%$ confidence intervals. $\dagger p<.10,{ }^{* *} p<.01,{ }^{* * *} p<.001$.

\begin{tabular}{lcc}
\hline Predictor of Incivility & $\begin{array}{c}\text { All Tweets } \\
(N=1,279,834)\end{array}$ & $\begin{array}{c}\text { Tweets about Trump Removed } \\
(N=1,213,071)\end{array}$ \\
All Democrats and Republicans & & \\
\hline Intercept) & $13.504[13.309,13.699]^{* * *}$ & $13.159[12.98,13.338]^{* * *}$ \\
Partisanship & $0.997[0.808,1.186]^{* * *}$ & $0.837[0.664,1.011]^{* * *}$ \\
Time & $0.285[0.272,0.298]^{* * *}$ & $0.192[0.179,0.205]^{* * *}$ \\
Party & $-1.207[-1.402,-1.012]^{* * *}$ & $-0.917[-1.096,-0.738]^{* * *}$ \\
Partisanship $\times$ Time & $0.039[0.025,0.052]^{* * *}$ & $0.014[0.000,0.027] \dagger$ \\
Partisanship $\times$ Party & $-0.363[-0.552,-0.174]^{* * *}$ & $-0.238[-0.412,-0.065]^{* *}$ \\
Time $\times$ Party & $-0.211[-0.224,-0.198]^{* * *}$ & $-0.141[-0.154,-0.128]^{* * *}$ \\
Partisanship $\times$ Time $\times$ Party & $-0.079[-0.093,-0.065]^{* * *}$ & $-0.059[-0.073,-0.046]^{* * *}$ \\
\hline Democrats & & $14.079[13.837,14.321]^{* * *}$ \\
(Intercept) & $14.713[14.427,14.999]^{* * *}$ & $1.076[0.829,1.324]^{* * *}$ \\
Partisanship & $0.496[0.473,0.518]^{* * *}$ & $0.333[0.311,0.355]^{* * *}$ \\
Time & $1.361[1.068,1.653]^{* * *}$ & $0.073[0.049,0.097]^{* * *}$ \\
Partisanship $\times$ Time & $0.118[0.093,0.142]^{* * *}$ & $12.24[11.98,12.501]^{* * *}$ \\
\hline Republicans & & $0.599[0.363,0.834]^{* * *}$ \\
(Intercept) & $0.12295[0.12029,0.12562]^{* * *}$ & \\
Partisanship & $0.00074[0.00059,0.00088]^{* * *}$ & \\
\hline
\end{tabular}




\begin{tabular}{lcc} 
Time & $0.00633[0.00392,0.00874]^{* * *}$ & $0.051[0.036,0.066]^{* * *}$ \\
Partisanship $\times$ Time & $-0.00041[-0.00055,-0.00026]^{* * *}$ & $-0.047[-0.061,-0.032]^{* * *}$ \\
& & \\
\hline $\begin{array}{l}\text { Moderate Democrats } \\
\text { Time }\end{array}$ & $0.260[0.238,0.282]^{* * *}$ \\
\hline $\begin{array}{l}\text { Liberal Democrats } \\
\text { Time }\end{array}$ & $0.406[0.366,0.447]^{* * *}$ \\
\hline Moderate Republicans & $0.614[0.573,0.655]^{* * *}$ & \\
Time & $0.114[0.091,0.138]^{* * *}$ & $0.097[0.074,0.121]^{* * *}$ \\
\hline Conservative Republicans & $0.033[0.016,0.050]^{* * *}$ & $0.004[-0.013,0.022]$ \\
Time &
\end{tabular}

Next, we tested whether the effects generalized across the House and Senate by conducting a factorial multilevel model predicting incivility with party, time, and chamber (and their interactions) as predictors. Table S3 shows how there was no effect of chamber, meaning that neither chamber was more uncivil than the other. We also found a null Party $\times$ Chamber interaction, meaning that the difference in levels of incivility between the parties was uniform across the two Chambers. We did, however, find a Time $\times$ Chamber interaction such that the rise in incivility was greater in the House than it was in the Senate. And a three-way interaction qualified that effect, meaning that the emergence of incivility from Democrats (compared to Republicans) over time has not been uniform in the two chambers.

To better understand the interaction, we ran Party $\times$ Time simple interaction models for each chamber (see Table S4) and found that Democrats' tweets were more uncivil than Republicans' in both chambers, incivility was on the rise in both chambers, and Democrats' greater incivility (relative to Republicans') grew over time in both chambers. These results suggest that incivility on Twitter has increased in both chambers of the U.S. Congress.

Table S4. Tests of whether there have been mean level changes in incivility among Democrats and Republicans over time, and whether the changes have differed between the House and Senate. The party variable was coded as -1 (Democrat) and 1 (Republican); Independents were excluded for this analysis. The time variable is in years, re-centered at the mean. The chamber variable was coded as -1 (House of Representatives) or 1 (Senate). ${ }^{* * *} p<.001$.

\begin{tabular}{|c|c|c|c|}
\hline & Omnibus & House & Senate \\
\hline (Intercept) & $13.225[12.911,13.539]^{* * *}$ & $13.339[13.137,13.541]^{* * *}$ & $13.114[12.658,13.571]^{* * *}$ \\
\hline Party & $-0.860[-1.174,-0.547]^{* * *}$ & $-0.739[-0.941,-0.537]^{* * *}$ & $-0.983[-1.439,-0.526]^{* * *}$ \\
\hline Time & $0.218[0.198,0.238]^{* * *}$ & $0.257[0.244,0.269]^{* * *}$ & $0.179[0.144,0.215]^{* * *}$ \\
\hline Chamber & $-0.115[-0.429,0.199]$ & & \\
\hline Party x Time & $-0.224[-0.243,-0.204]^{* * *}$ & $-0.183[-0.195,-0.170]^{* * *}$ & $-0.264[-0.300,-0.229]^{* * *}$ \\
\hline Party x Chamber & $-0.121[-0.435,0.192]$ & & \\
\hline Time $\mathrm{x}$ Chamber & $-0.039[-0.059,-0.019]^{* * *}$ & & \\
\hline Party $\mathrm{x}$ Time $\mathrm{x}$ Chamber & $-0.041[-0.061,-0.021]^{* * *}$ & & \\
\hline
\end{tabular}

Covariates. We find that the rise in incivility over time holds up even when controlling for various demographic, political, and linguistic covariates, each of which may have shifted 
over time and could have been confounding variables. Covariates included demographics factors such as the gender, race, and age of the politicians. Political considerations included the degree to which the politician was a partisan versus a moderate, and the degree to which their home district was electorally uncontentious and therefore safe. Linguistic factors included the overall emotional tone of the tweet, the degree to which the tweet expressed moral outrage, and whether or not the tweet mentioned President Trump.

Demographic Covariates. If certain demographic groups' tweets are scored higher on incivility (either due to some invalid bias and/or for more valid reasons), this could account for the observed rise in incivility. We tested this shifting-demographics confound by testing whether controlling for the effect of gender $(1=$ female, $0=$ male $)$, race $(1=$ black, $0=$ non-black $)$, and age (z-scores) in three separate models reduced the rate of increase of incivility over time.

Gender and race did not help account for the increase. However, age did to a small extent, with older members' tweets scoring higher on incivility than those of younger members, $\mathrm{B}=1.9,95 \%$ $\mathrm{CI}=[1.8,2.0], p<.001$, and the average age of members of Congress increasing over time, $\mathrm{B}=$ $3.1,95 \% \mathrm{CI}=[3.0,3.2], p<.001$. Thus, the greying of Congress partially accounted for the rise of incivility (the incivility-time slope was reduced by 17\%).

Political Covariates. Political polarization has increased in recent decades, with Democrats shifting to the political left and Republicans shifting toward the political right in their policy positions (Boxell et al., 2017). Rising ideological distance between the two parties and increasing district safety could help account for the observed rise in incivility. We tested this political polarization account by controlling for the effect of partisanship of members of Congress ( $\mathrm{z}$ scores of absolute values of DW-Nominate 1 scores) and district safety in the most recent midterm election (operationalized as the share of the popular vote, standardized) and observing whether the rate of change of incivility over time was diminished in two separate analyses. No such changes were observed (see Figure 2), meaning that political polarization appears to not account for the rise in incivility.

Linguistic Covariates. The rise in incivility may be a by-product of a general decline in the emotional tone of language (perhaps due to rising sadness or anxiety) and/or a rise in moral outrage. Although it might seem that incivility and emotional tone would be strongly correlated, their correlation ( $r=-.40$ in tweets) is far from the conventional multicollinearity threshold of $r=$ .80. And moral outrage and incivility were only correlated at $r=.17$, meaning that there was substantial independence (both conceptually and empirically) between incivility and moral outrage/emotional tone. We therefore tested whether general changes in emotional tone or specific changes in moral outrage account for the observed rise in incivility over time. Paradoxically, while moral outrage increased over time, $\mathrm{B}=3.79,95 \% \mathrm{CI}=[3.73,3.85], p<$ .001 , so did the overall positivity of the emotional tone, $\mathrm{B}=1.65,95 \% \mathrm{CI}=[1.60,1.71], p<$ .001 . Holding emotional tone constant in a multilevel model made the rise in incivility even steeper. That is, the rise in incivility was observed in spite of a generally more positive emotional tone of tweets over time. Holding moral outrage constant in a multilevel model made the rise in incivility approximately $43 \%$ less steep, meaning that the rise of moral outrage partly, but not fully, accounted for the rise in incivility.

As we will observe and discuss in Study S5, President Trump's tweets were more uncivil than those of his predecessor and became more uncivil during his first three years in office. Members of Congress may have responded to the President Trump's tweeting, statements, and actions with incivility of their own. To test the possibility that reactions to President Trump account for the rise in incivility, we classified tweets by members of U.S. Congress as either mentioning 
President Trump or not by creating a LIWC dictionary with the stems Trump*, Donald*, @ realdonaldtrump, and \#Trump* and used it to code tweets dichotomously, as either having (1) or not having (0) any mention of President Trump. Trump was mentioned in $5.25 \%$ of tweets (see Table S5 for a breakdown by year).

Table S5. Number of tweets that mentioned Donald Trump by year.

\begin{tabular}{lrrr}
\hline Year & $\begin{array}{r}\text { Trump mentioned? (ntweets) } \\
\text { Trump not } \\
\text { mentioned }\end{array}$ & $\begin{array}{r}\text { Trump } \\
\text { mentioned }\end{array}$ & $\begin{array}{r}\text { \% Trump } \\
\text { mentioned }\end{array}$ \\
\hline 2009 & 7,702 & 3 & $0.04 \%$ \\
2010 & 19,890 & 8 & $0.04 \%$ \\
2011 & 35,941 & 7 & $0.02 \%$ \\
2012 & 49,895 & 39 & $0.08 \%$ \\
2013 & 74,260 & 17 & $0.02 \%$ \\
2014 & 98,974 & 35 & $0.04 \%$ \\
2015 & 121,568 & 283 & $0.23 \%$ \\
2016 & 153,924 & 2,866 & $1.83 \%$ \\
2017 & 224,552 & 20,741 & $8.46 \%$ \\
2018 & 256,661 & 25,521 & $9.04 \%$ \\
2019 & 181,236 & 18,305 & $9.17 \%$ \\
\hline
\end{tabular}

If the rise in incivility was attributable to reactions to President Trump, we should expect to find that tweets mentioning President Trump were more uncivil $(M=21.1, S D=13.2)$ than those that did not mention $\operatorname{him}(M=13.1, S D=9.8)$, and this was confirmed, $B=6.0,95 \% \mathrm{CI}=[6.0,6.1]$, $p<.001$. Controlling for mentioning Trump reduced the incivility-time slope by $32 \%$ meaning that reactions to Trump helped but did not fully account for the observed rise in incivility.

Reinforcement learning explains the rise in incivility over time. Uncivil tweets were received differently on Twitter than civil ones, with uncivil tweets receiving more "retweets" and more "likes" than civil ones. This outsized reaction might have communicated to the tweet author that their incivility was well-received and thus operantly reinforced the original uncivil behavior. We tested whether uncivil tweets received more "retweets" and "likes" than civil ones. Finding that the "likes" and "retweets" metric evidenced skewed distributions, we log-transformed them prior to analyses. Multilevel models, with random intercepts for each Twitter account, found that uncivil tweets received 10 times more "retweets" and 8 times more "likes" than civil ones (see Table S6). The same pattern was found when including tweets by sitting members only. For all subsequent analyses, we conservatively use the full data set.

Table S6. The effect of incivility on the three metrics ("retweets", "likes", and "likes"-per-"retweet") from multilevel analyses. In each analysis, there was a single predictor (i.e. incivility) of a single outcome (e.g., "retweet" count). All metrics were log-transformed for these analyses. Numbers represent order of magnitude increases resulting from incivility. ${ }^{* * *} p<.001$

\begin{tabular}{lcc}
\hline & \multicolumn{2}{c}{ Incivility as Predictor of Outcome } \\
\cline { 2 - 3 } Outcome & $\begin{array}{c}\text { All Members } \\
\left(N_{\text {tweets }}=1,284,124\right)\end{array}$ & $\begin{array}{c}\text { Sitting Members Only } \\
\left(N_{t w e e t s}=1,129,930\right)\end{array}$ \\
\hline "Retweets" & $.01024[.01012, .01036]^{* * *}$ & $.01072[.01059, .01085]^{* * *}$ \\
"Likes" & $.00917[.00904, .00931]^{* * *}$ & $.00950[.00936, .00964]^{* * *}$
\end{tabular}


The finding that uncivil tweets receive more "likes" and "retweets" seems to imply that uncivil tweets were well received on Twitter. As we show, the validity of the "likes" metric is poor in that the more the Twitter community dislikes a tweet, the more "likes" it receives (because the greater number of "retweets" of uncivil tweets drives up the exposure of uncivil tweets; see Studies S2-3 for a full explanation). A "likes"-per-"retweet" metric might be a valid metric of sentiment because it is not confounded with exposure and tracks positively with approval (in both on- and off-Twitter samples; see Studies S2-3). Analyses with the "likes"-per-"retweet" (approval) metric supports a different conclusion about whether uncivil tweets were genuinely well-received: Uncivil tweets received 22\% fewer "likes"-per-"retweet" as civil ones (see Table S5). This finding suggests that despite the elevated "likes" count, the Twitter community actually disapproved of uncivil tweets.

Rising reactivity to incivility. If the outsized reaction to uncivil tweets has grown over time, then current members are receiving even more "likes" and "retweets" for their uncivil tweets than they used to (relative to their civil ones). We find support for this notion that the incivility"retweet" and incivility-"likes" relationships have strengthened over time. The time variable in this analysis was in years and re-centered at the mean (2016.81, which was in September 2016). We entered time, incivility, and their interaction into a multilevel model (random intercepts for each Twitter handle), predicting the number of "retweets". And we did the same with the number of "likes" as the dependent variable.

Table S7 and Figure 4 shows how reactions to incivility have generally become stronger over time. Uncivil tweets (incivility $=100$ ) received 1.66 times as many "retweets" as civil ones (incivility = 0) in 2009 but 14.73 times as many "retweets" than civil ones in 2019-a 9-fold increase in reactivity. And uncivil tweets received 1.29 times as many "likes" as civil tweets in 2009 but 7.88 times as many "retweets" as civil ones in 2019-a 6-fold increase in reactivity. These results suggest that the reactions to incivility (in terms of elevated "retweets" and "likes") are a recent development. For the "likes"-per-"retweet" ratio, the overall trend has been that uncivil tweets receiving fewer "likes"-per-"retweet" than civil ones, implying that the Twitter community does not approve of incivility. However, this ratio has weakened slightly over time, meaning that aversion for incivility is weakening over time.

Table S7. Reactions, in terms of elevated "liking" and "retweeting," to incivility have strengthened over time. Numbers represent the unstandardized effects of incivility, time, and their interaction on various metrics. Time is in years and recentered at the mean. Each metric is log-transformed. Numbers in brackets represent $95 \%$ confidence intervals. ${ }^{* * *} p<.001$

\begin{tabular}{lccc}
\hline & & Effect of Predictor on... & \\
\cline { 2 - 4 } Predictor & \multicolumn{1}{c}{ "Retweets" } & "Likes" & \multicolumn{1}{c}{$\begin{array}{c}\text { "Likes"-per-"Retweet" } \\
\text { (Approval) }\end{array}$} \\
\hline Incivility & $.00936[.00925, .00948]^{* * *}$ & $.00748[.00737, .00759]^{* * *}$ & $-.00189[-.00198,-.00180]^{* * *}$ \\
Time (years) & $.120[.119, .121]^{* * *}$ & $.279[.278, .280]^{* * *}$ & $.159[.158, .160]^{* * *}$ \\
Incivility $\times$ Time & $.00093[.00088, .00099]^{* * *}$ & $.00105[.00100, .00110]^{* * *}$ & $.00012[.00008, .00016]^{* * *}$ \\
\hline
\end{tabular}

To test whether this increasing reactivity helps explain the rise in incivility, we calculated the reactivity (incivility-"retweets" slope, incivility-"likes" slope) for each political party in each year (e.g., Republicans in 2013). We then tested whether statistically controlling for this increasing reactivity has the effect of diminishing or eliminating the rise in incivility over time. We found that the "likes" metric explained $74 \%$ of the rise, and in a parallel analysis, the 
"retweets" metric fully explained (and even reversed) the rise over time (see Figure S1). Thus, Twitter metric reinforcement explained the observed rise in incivility over time.

Granger causality. The previous analysis showed that statistically controlling for increasingly strong reactions to uncivil tweets had the effect of blunting or eliminating the observed rise in incivility over time. The correlational nature of this statistical correction leaves open questions about whether the reaction precipitated the rise. The longitudinal nature of these data allows us to examine whether earlier experiences with uncivil tweeting predicted subsequent changes in incivility on the part of the tweeter. To illustrate the concept of this analysis, we use the "likes" metric; the same logic applies to the "retweets" metric.

The reaction to incivility tended to be positive on average, meaning that uncivil tweets received more "likes" than civil ones. However, we observe variation in the degree to which uncivil tweets were "liked" more often than civil ones. The histogram in Figure S1 shows that, for the modal tweet, there was a positive association between incivility and the number of "likes." However, there was a sizable number of times that members of Congress' civil and uncivil tweets received similar numbers of "likes" ("likes"-incivility slope $=0$ ). And there were a sizable but smaller number of instances in which uncivil tweet received many more "likes" than civil ones. 
Figure S1. Histogram of the slope of (log-transformed) "likes" to the level of incivility in the 50 most recent tweets by members of Congress. The "likes" metric being log-transformed means that a value of 0 means no effect and a value of .01 means a 10 -fold increase for uncivil (AI score $=100$ ) versus civil $(\mathrm{AI}$ score $=0)$ tweets $\left(=10^{100 \times .01}\right)$. The mode being around .0075 means that at the modal time, uncivil tweets received 5.6 times more "likes" than civil ones $\left(=10^{100 \times .0075}\right)$.

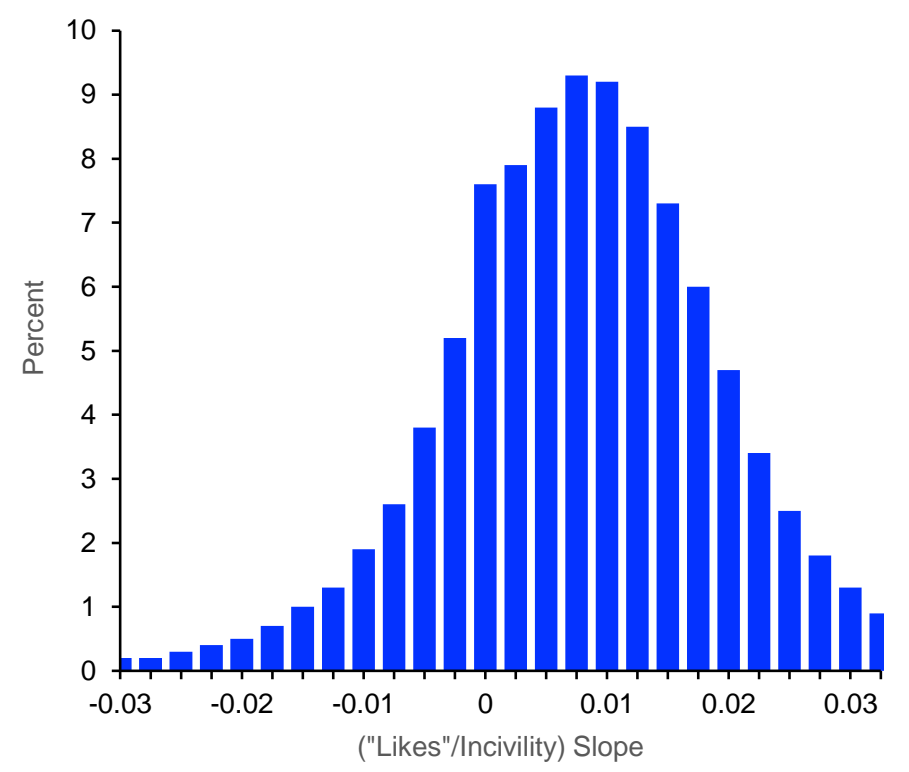

If members of Congress learned from metrics, then during times when uncivil tweets received a particularly large number of "likes" compared to civil tweets, the tweeter would have received strong reinforcement, causing the politician to become even more uncivil in the next set of tweets. In contrast, during times when civil and uncivil tweets received the same number of "likes", the tweeter would have received no reinforcement for their behavior, and there should be no change in the level of incivility of the next set of tweets. Formally, therefore, variations in the slope of incivility to "liking" in recent history should positively predict subsequent levels of incivility. Figure S2 illustrates the concept that our analyses aim to formalize. 
Figure S2. Conceptual model for inferring whether Twitter metrics reinforced uncivil tweeting. If metrics (e.g., the number of "likes" resulting from an uncivil tweet) has a reinforcing effect, then during times when uncivil tweets receive a lot more "likes" than civil ones, we should expect an uptick in incivility in subsequent tweets. But when uncivil tweets are not liked more than civil ones, we should expect less of an uptick in the incivility of future tweets.

\section{Last 50 Tweets}

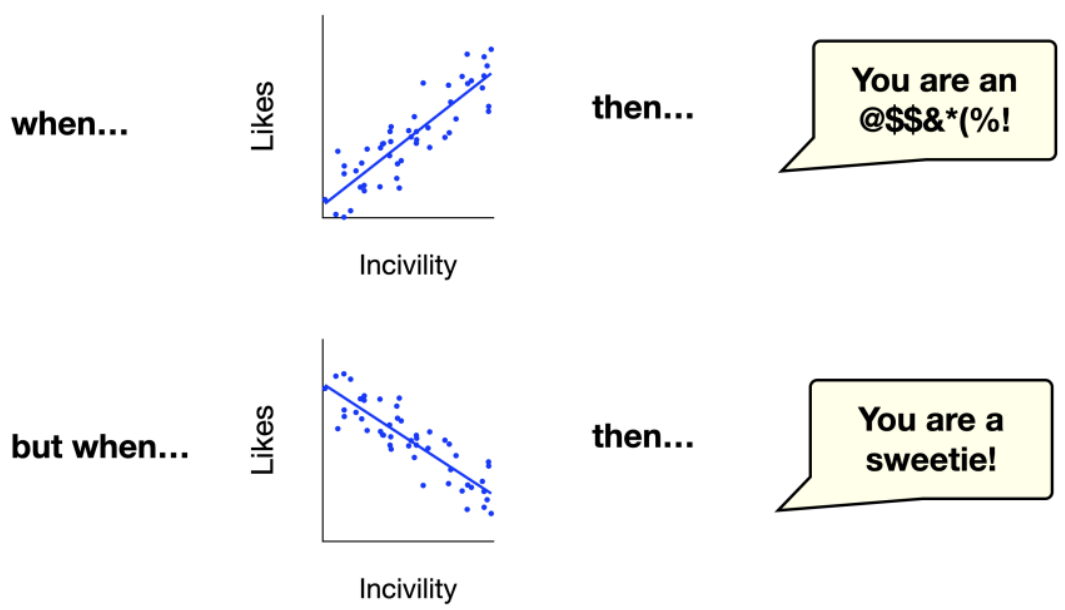

We chose to use a 50-tweet window to balance competing considerations. One consideration is human memory. It is unlikely that reactions to a tweet that happened 100 or 200 tweets ago would have much if any effect on current behavior insofar as it would have faded from memory. Thus, memory limitations motivate the use of a shorter tweet window. The competing consideration is statistical. Longer windows produce more stable slope and mean estimates. If estimates are unstable, then there will be a relatively large number of outliers in our analyses, violating assumptions in statistical models, and potentially having an outsized influence on our results. Even a window of 50 tweets yielded distributions that were skewed (see Table S7. Examination of the histograms revealed that these skews were the result of a few outliers (382 out of 1.2 million, which were more than 7 SDs from the mean). Excluding these $0.03 \%$ of observations yielded normal distributions (see Table S8). The window of 50, along with a few exclusions, strikes a balance between empirical considerations regarding normality and the limits of human memory.

Table S8. Descriptive statistics of learning analysis variables, both with and without outliers.

\begin{tabular}{|c|c|c|c|c|c|c|}
\hline \multirow[b]{2}{*}{ Metric } & \multicolumn{3}{|c|}{ With Outliers $(N=1,212,955)$} & \multicolumn{3}{|c|}{ Without Outliers $(N=1,212,573)$} \\
\hline & $M(S D)$ & Range & Skew & $M(S D)$ & Range & Skew \\
\hline Likes: Incivility Slope & $-0.02(11.43)$ & -6222.40 to 6.93 & -490.13 & $0.0070(0.0123)$ & -0.099 to 0.10 & 0.00 \\
\hline Retweets: Incivility Slope & $0.20(85.16)$ & -0.33 to 40346.72 & 450.14 & $0.0088(0.0131)$ & -0.099 to 0.10 & -0.06 \\
\hline Incivility & $14(4)$ & 2 to 48 & 1.55 & $14(4)$ & 2 to 48 & 1.54 \\
\hline Future Incivility & $14(4)$ & 2 to 48 & 1.53 & $14(4)$ & 2 to 48 & 1.53 \\
\hline
\end{tabular}

Our Granger analyses found evidence of autocorrelation (coefficient $a$ ), and that after controlling for autocorrelation, "retweet" and "likes" metrics had a reinforcing effect on incivility (coefficient $b$; see Table S9). Following times that uncivil tweets received a particularly large 
number of "likes" and "retweets" relative to civil tweets, rises in subsequent uncivil tweeting were the steepest.

Table S9. Politicians learned from their uncivil tweets receiving many "likes" and "retweets". Analyses were Granger correlational analyses in which the relationship (slope) between incivility and the resulting number of "likes" and "retweets" the tweet received (in the 50 most recent tweets) predicted how uncivil the next 50 tweets would be, controlling for how uncivil the 50 most recent tweets were. Analyses were multilevel models with random intercepts for each politician. Coefficients $b$ in the table thus represent the unstandardized effects of learning from recent events. The positive effects indicate that "likes" and "retweets" metrics had a rewarding effect on incivility. Numbers in brackets are $95 \%$ confidence intervals; ${ }^{* * *} p<.001$

\begin{tabular}{lcc}
\hline Predictors of Later Incivility & Likes & Retweets \\
\hline (Intercept) & $9.141[8.996,9.286]^{* * *}$ & $9.124[8.980,9.269]^{* * *}$ \\
a, Earlier Incivility (autocorrelation) & $0.315[0.313,0.317]^{* * *}$ & $0.315[0.313,0.317]^{* * *}$ \\
$b$, Earlier Incivility-Metric Slope & $3.513[3.204,3.821]^{* * *}$ & $4.336[4.047,4.625]^{* * *}$ \\
\hline
\end{tabular}

The 50-tweet window in the prior analysis is somewhat arbitrary. We therefore conducted similar analyses with 30- and 80-tweet windows. We removed metric-incivility outliers (more than 3 $S D$ s from the $M \mathrm{~s}$ ), resulting in retention rates of $98.77 \%$ and $98.85 \%$ for the 30 -tweet window analyses ("likes" and "retweets" analyses respectively) and $99.06 \%$ and $99.94 \%$ retention for the 80-tweet window. Table S10 shows how similar Granger causal effects were found when using 30- and 80-tweet windows.

Table S10. Granger causality analyses with 30 -tweet and 80-tweet windows.

\begin{tabular}{|c|c|c|c|c|}
\hline \multirow[b]{2}{*}{$\begin{array}{l}\text { Predictors of Later } \\
\text { Incivility }\end{array}$} & \multicolumn{2}{|c|}{30 Tweet Window } & \multicolumn{2}{|c|}{80 Tweet Window } \\
\hline & Likes & Retweets & Likes & Retweets \\
\hline (Intercept) & $9.982[9.826,10.138]^{* * *}$ & $8.461[8.325,8.597]^{* * *}$ & $9.952[9.797,10.108]^{* * *}$ & $8.471[8.335,8.608]^{* * *}$ \\
\hline $\begin{array}{l}\text { a, Earlier Incivility } \\
\text { (autocorrelation) }\end{array}$ & $0.252[0.251,0.254]^{* * *}$ & $0.252[0.251,0.254]^{* * *}$ & $0.252[0.251,0.254]^{* * *}$ & $0.252[0.251,0.254]^{* * *}$ \\
\hline $\begin{array}{l}b \text {, Earlier Incivility- } \\
\text { Metric Slope }\end{array}$ & $2.96[2.66,3.26]^{* * *}$ & $7.32[6.97,7.67]^{* * *}$ & $3.27[3.00,3.55]^{* * *}$ & $6.82[6.51,7.13]^{* * *}$ \\
\hline
\end{tabular}

\section{Summary}

We found that among members of the U.S. Congress, incivility increased over time. These effects generalized across the political spectrum and generalized to both the House and Senate. Various covariates did not substantially explain these trends. In contrast, the tendency for uncivil tweets to receive many more "retweets" and "likes" than civil tweets did explain the observed change over time in both mediational and longitudinal analyses.

\section{Presidential Tweets}

We tested whether presidential incivility increased over time, and whether the rate of change differed between the two presidents (see Table S11 for descriptive statistics). For this analysis, the time variable was in units of years and centered at the beginning of 2019. An OLS regression analysis with Time, the President $(1=$ Trump, $-1=$ Obama $)$, and their interaction, predicting incivility, yielded a positive effect of Time, $\mathrm{B}=0.9,95 \% \mathrm{CI}=[0.8,1.1], \beta=.195, p<.001$, consistent with the idea that presidential incivility increased over time on Twitter. We also found 
a main effect of President, $\mathrm{B}=4.5,95 \% \mathrm{CI}=[4.0,4.9], \beta=.340, p<.001$; President Trump's tweets were more uncivil than those of President Obama. And we found an interaction, $\mathrm{B}=1.0$, $95 \% \mathrm{CI}=[0.8,1.2], \beta=.225, p<.001$. The rise in incivility over time was greater for President Trump, $\mathrm{B}=1.9,95 \% \mathrm{CI}=[1.6,2.3], \beta=.101, p<.001$, than it was for President Obama, $\mathrm{B}=-$ $0.1,95 \% \mathrm{CI}=[-0.2,0.0], \beta=-.013, p=.231$.

Table S11. Descriptive statistics of the tweets of by Presidents Obama and Trump ( $N=17,483$ tweets)

\begin{tabular}{lrrrrrr}
\hline & \multicolumn{3}{c}{ President Obama } & \multicolumn{3}{c}{ President Trump } \\
& $M$ & \multicolumn{1}{c}{$S D$} & Skew & $M$ & $S D$ & Skew \\
\hline Raw Metrics & & & & & & \\
"Retweets" & 1,450 & 10,958 & 62.131 & 20,298 & 11,923 & 6.622 \\
"Likes" & 1,718 & 8,837 & 44.091 & 86,340 & 43,124 & 3.012 \\
"Likes"-per-"retweet" & 4.918 & 47.162 & 19.235 & 4.362 & 0.766 & 1.590 \\
$\quad$ Incivility & 13.4 & 7.9 & 1.824 & 21.5 & 15.5 & 1.127 \\
Log-Transformed & & & & & & \\
"Retweets" & 2.761 & 0.609 & -1.927 & 4.242 & 0.289 & -3.920 \\
$\quad$ "Likes" & 2.769 & 0.626 & -0.175 & 4.874 & 0.313 & -6.200 \\
"Likes"-per-"retweet" & 0.009 & 0.505 & 0.929 & & & \\
\hline
\end{tabular}

\section{Study S1: Validity of PerspectiveAPI}

Does PerspectiveAPI's incivility-scoring algorithm really anticipate whether people will perceive a political tweet to be uncivil? Although it was trained on a large set of annotated texts, its validity remains to be established publicly and independently.

\section{Method}

Participants. We recruited roughly equal numbers of Democrats and Republicans on TurkPrime, a crowdsourcing website, using TurkPrime's pre-screened party identification measure. The final sample $(N=160)$ included 71 Republicans, 16 Independents, and 73 Democrats. The sample was $61 \%$ male, $70 \%$ white, and 38 years old on average $(S D=13)$. The majority $(79 \%)$ reported that they are on Twitter. Each participant received $\$ 1.00$.

Procedure. Participants reviewed 20 randomly selected tweets (out of 40) and rated how polite, positive, and opinion-based each tweet was. Collectively the participants made 9,597 judgments. They then indicated how they would feel about their own tweets receiving various responses (to assess how people respond to metrics; not analyzed here), reported demographics, and were debriefed.

Tweets. The sample of tweets was from sitting Democrats and Republicans (not Independents) that represented the full spectrum of incivility. We coded the level of incivility of each tweet in Study 1 using PerspectiveAPI then randomly selected original tweets (no retweets) that had an incivility score of $0,5,10, \ldots, 85,90,95$. Thus, there were 20 tweets by Democrats and 20 tweets by Republicans. We removed URLs and emojis. To avoid overtaxing participants, we asked them to read and respond to a random sampling of 20 of the 40 tweets, in random order. 
They saw only the text of the tweet. They were not made aware of the name or party of the tweeter or provided any context. The tweets are shown in Table S12.

Table S12. The tweets that participants judged in Study S1.

\begin{tabular}{|c|c|c|c|}
\hline Tweeter & Date & Text & Incivility \\
\hline John Garamendi (D) & 12 June 2019 & $\begin{array}{l}\text { Right now the Chairman of the Committee, Adam Smith (D- } \\
\text { WA), is providing an overview of the process and delivering his } \\
\text { introductory remarks. }\end{array}$ & 0 \\
\hline Rep. Anna G. Eshoo (D) & 11 September 2017 & $\begin{array}{l}\text { Today and every day we remember and honor those who } \\
\text { demonstrated valor and courage, from the heroes on Flight } 93 . .\end{array}$ & 5 \\
\hline Denny Heck (D) & 16 May 2018 & $\begin{array}{l}\text { Can you imagine being denied a financial protection because you } \\
\text { don't have the right paperwork? Our active duty servicemembers } \\
\text { deserve better. I'm asking Congress to update the law so this } \\
\text { stops happening. }\end{array}$ & 10 \\
\hline Mark DeSaulnier (D) & 25 February 2018 & $\begin{array}{l}\text { Talked to @nbcbayarea about Congress' inaction on gun violence } \\
\text { and the need for an open debate and vote to help prevent future } \\
\text { tragedies. }\end{array}$ & 15 \\
\hline Anthony G. Brown (D) & 05 April 2017 & $\begin{array}{l}\text { Judge Gorsuch has consistently ruled against minorities, women, } \\
\text { people with disabilities \& workers. He isn't in the mainstream }\end{array}$ & 20 \\
\hline Rep. Gil Cisneros (D) & 12 June 2019 & $\begin{array}{l}\text { Today marks } 3 \text { years since the tragic shooting at \#Pulse Nightclub } \\
\text { in Orlando. Today, I join my colleagues, constituents, \& fellow } \\
\text { Americans in remembering the victims of this massacre as we } \\
\text { continue to fight for love \& acceptance for all. \#LGBTQ }\end{array}$ & 25 \\
\hline Rep. Ilhan Omar (D) & 05 February 2019 & $\begin{array}{l}\text { I will not bow down to hate or bigotry. I will not allow others to } \\
\text { treat me as a 2nd class citizen. I will stand strong with you as we } \\
\text { fight to protect all Americans in every community, no matter } \\
\text { their religion, race, ethnicity, sexual orientation or gender } \\
\text { identity. }\end{array}$ & 30 \\
\hline Rep. Joe Kennedy III (D) & 04 September 2017 & $\begin{array}{l}\text { It's a cruel hand that extends the American dream and then steals } \\
\text { it away. \#DACA \#DREAMers }\end{array}$ & 35 \\
\hline Chris Van Hollen (D) & 14 September 2016 & $\begin{array}{l}\text { We have a bipartisan \#NoFlyNoBuy bill to stop suspected } \\
\text { terrorists from buying guns. @SpeakerRyan, let us vote! } \\
\text { \#DoYourJob \#CloseTheLoophole }\end{array}$ & 40 \\
\hline Dina Titus (D) & 11 March 2019 & $\begin{array}{l}\text { By proposing that even more money be wasted on Yucca } \\
\text { Mountain, Trump has broken the promise he made to Nevadans } \\
\text { during his recent visit to Elko. Every dollar this Administration } \\
\text { tries to put towards the revitalization of Yucca Mountain is } \\
\text { another dollar down a rat hole... }\end{array}$ & 45 \\
\hline $\begin{array}{l}\text { Rep. Bonnie Watson } \\
\text { Coleman (D) }\end{array}$ & 06 July 2018 & $\begin{array}{l}\text { So much for 'civility.' President's continued attacks on Rep. } \\
\text { Waters are simply pathetic. She's a tireless leader who fights for } \\
\text { the American people every day. Ultimately POTUS goes on these } \\
\text { mindless rants to distract from unpopular policies, crony cabinet, } \\
\text { and mounting scandals }\end{array}$ & 50 \\
\hline Cicilline Press Office (D) & 20 June 2017 & $\begin{array}{l}\text { It's time for Republicans to \#ShowUsTheBill and stop trying to } \\
\text { hide \#Trumpcare from the American people! }\end{array}$ & 55 \\
\hline Hakeem Jeffries (D) & 01 May 2018 & $\begin{array}{l}\text { The American people deserve to know whether or not the } \\
\text { President is a crook \#FollowTheFacts }\end{array}$ & 60 \\
\hline Ami Bera, M.D. (D) & 27 April 2017 & $\begin{array}{l}\text { Majority's plan punishes old, sick, \& kicks millions off health } \\
\text { care. As a doctor, I'm going to fight this reckless bill. } \\
\text { \#ProtectOurCare }\end{array}$ & 65 \\
\hline Jan Schakowsky (D) & 28 May 2018 & $\begin{array}{l}\text { Children are being cruelly ripped away from their mothers } \\
\text { because of YOUR policies Mr. President. The American people } \\
\text { can see through your shameless lies. }\end{array}$ & 70 \\
\hline US Rep. Filemon Vela (D) & 26 January 2017 & $\begin{array}{l}\text { @POTUS continues to claim that @mexico will pay for his } \\
\text { ridiculous border wall. \#TheBigLie \#WALLternativefacts }\end{array}$ & 75 \\
\hline Rep. Alan Grayson (D) & 10 April 2015 & $\begin{array}{l}\text { Cheney, you nearly bankrupted the US, and you have blood on } \\
\text { your hands. Slither back into your hole. }\end{array}$ & 80 \\
\hline Rep. John Yarmuth (D) & 24 June 2019 & $\begin{array}{l}\text { Hey moron: the third line of the Iran Nuclear Deal guaranteed } \\
\text { just that. Maybe you should have read it before you tore it up. }\end{array}$ & 85 \\
\hline
\end{tabular}


US Rep. Filemon Vela (D)

26 November 2017

US Rep. Filemon Vela (D)

11 April 2017

Sen. Lisa Murkowski (R)

30 May 2019

Eric Cantor (R)

Justin Amash (R)

Judge Carter (R)

Ted Poe (R)

Raúl R. Labrador (R)

Kevin McCarthy (R)

Dr. Phil Roe (R)

Lindsey Graham (R)

Rep. Jody Hice (R)

Brad Wenstrup (R)

Rep. Doug Collins (R)

Dana Rohrabacher (R)

Diane Black (R)

Tim Huelskamp (R)

Rep. Don Bacon (R)

Lindsey Graham (R)

Dana Rohrabacher (R)

Dan Bishop (R)

Dana Rohrabacher (R)
20 December 2013

27 October 2017

04 March 2015

19 June 2017

01 September 2015

11 June 2019

19 March 2013

03 September 2013

24 October 2016

28 April 2019

19 April 2019

25 August 2015

22 October 2015

10 September 2015

11 March 2019

01 August 2012

08 April 2015

10 July 2019

12 June 2016 @washingtonpost What an idiot... Does @realDonaldTrump

foolishly think a border wall would have prevented 9/11,

Charleston, San Bernardino, Orlando, Las Vegas, and Sutherland Springs?

Spicer on Hitler... What an idiot

96

A dedicated professional who exemplified Southern hospitality, Thad dedicated his life to his country and his family, and was an inspiration to many. My condolences to the Cochran family and their friends as they grieve the loss and celebrate the life of our former Senator.

Many Americans had good health care that they liked and could afford, but lost it due to ObamaCare.

Government spending keeps going up. What happened to fiscal 10 conservatism? Join me to discuss this and other topics.

7.5 million people will lose healthcare subsidies thanks to

Obamacare. Its time for a full repeal of \#Obamacare!

Our goal is to break the cycle of exploitation while restoring the lives of these innocent women and children....

A history of mistrust, a risky agreement, false imprisonment: I'll 25 be voting NO on the Iran nuclear deal.

Chinese threats to restrict rare earth minerals should be a wakeup call. These resources are essential to America's technology and defense, and we shouldn't have to depend on China.

The biggest problem $w$ healthcare in this country is cost. This 35

law does nothing to address that. \#FWRoundtable

This is the most illogical military operation I have ever seen - $\quad 40$ ramping up for war then backing off.

More proof that \#Obamacare is a complete and utter disaster. It's clear that this disastrous law is beyond repair.

I stand with members of the Jewish community, especially during this Holocaust Remembrance Week. The shooting yesterday in a synagogue in Poway, California is abhorrent. Hate and anti-Semitism have no place in our world.

This is ridiculous. AG Barr gives Democrats unprecedented accommodations, and they refuse them hours after Chairman

Nadler subpoenas those same answers. They've had 30 hours with the \#MuellerReport and realize the more information they actually get, the more bitter it tastes.

@DumbSci if Someone promotes the arrogant lie that $97 \%$ of all scientists agree with AGW theory, then I guess that person lacks credibility

As a military wife \& mother I am appalled. @POTUS veto of \#FY16NDAA puts our national security \& our troops at risk. Shameful.

This is no deal. It is a gift to Iran. It is a gift to radical Islam. It is a gift to all those who chant Death to America. \#IranDeal @PaulHammelOWH @GovRicketts Those comments were absolutely vile and repugnant.

Sequestration is Latin for 'dumb politicians destroying defense.'

@javalinarage those who claim that those who disagree must be stupid or corrupt are talking about themselves

Dumb. Dangerous. Reckless. What say you @McCreadyForNC? 90

@DriveLikeGW my how intellectual of U. Guess U R so superior 94 $\mathrm{U}$ can call those with whom $\mathrm{U}$ disagree assholes \& still think highly of Urself.

Judgments. Participants judged each tweet along three dimensions. First, they rated how polite each tweet was. Below the tweet text, a question asked, "How polite is it?" Reponses were on a 
7-point scale anchored at -3 (extremely rude), -2 (moderately rude), -1 (slightly rude), 0 (neutral), 1 (slightly polite), 2 (moderately polite), and 3 (extremely polite). Agreement among judges was high $(\mathrm{ICC}=.85)$. The second question assessed the overall tone of the tweet by asking, "How positive is the tone?" Responses were on a similar 7-point scale with the word rude replaced with negative and polite replaced with positive. The third question assessed how opinion-based the tweet was. The question asked, "Is it more opinion or fact based?" Responses were on a similar scale, ranging from extremely fact-based to extremely opinion based. We linearly scaled each measure so that they could range from 0 to 100 (with the -3 anchor becoming 0 and the +3 anchor becoming 100).

Demographics. Along with age, gender, and race, participants reported their party identification (Democrat, Independent, Republican), ideology (extremely liberal to extremely conservative on a 7-point scale), and whether they were on Twitter.

\section{Results}

Each participant judged 20 tweets. We modeled the relationship between human- and AI-scored incivility with a multilevel model that included random intercepts for each human judge. We then tested whether human-scored politeness correlated with AI-scored incivility and found that it did in the manner predicted, $\mathrm{B}=-0.599,95 \%$ confidence interval $=[-0.627,-0.571], p<.001$. To estimate an effect size, we standardized (z-scored) human-scored politeness and AI-scored incivility, yielding $\beta=-.627$. This result suggests that PerspectiveAPI accurately anticipates the reactions of humans.

To test whether this accuracy was limited to Twitter users of a particular party, judges of a particular party, both, or their combination, we included the Twitter user's party and the judge's party (-1 = Democrat, 1 = Republican) in a full factorial model (we excluded Independents for this analysis). The only significant predictor of AI-scored incivility in this model was judged politeness (see Table S13), meaning that the accuracy of AI-scored incivility at anticipating human judgments does not appear to be limited to or contingent upon the partisanship of either the tweeter or the judge. 
Figure S3. Average politeness rating across all Democratic judges (left panel) and Republican judges (right panel) of 20 tweets by Democratic and 20 tweets by Republican politicians that varied in how they were scored for incivility by PerspectiveAPI.
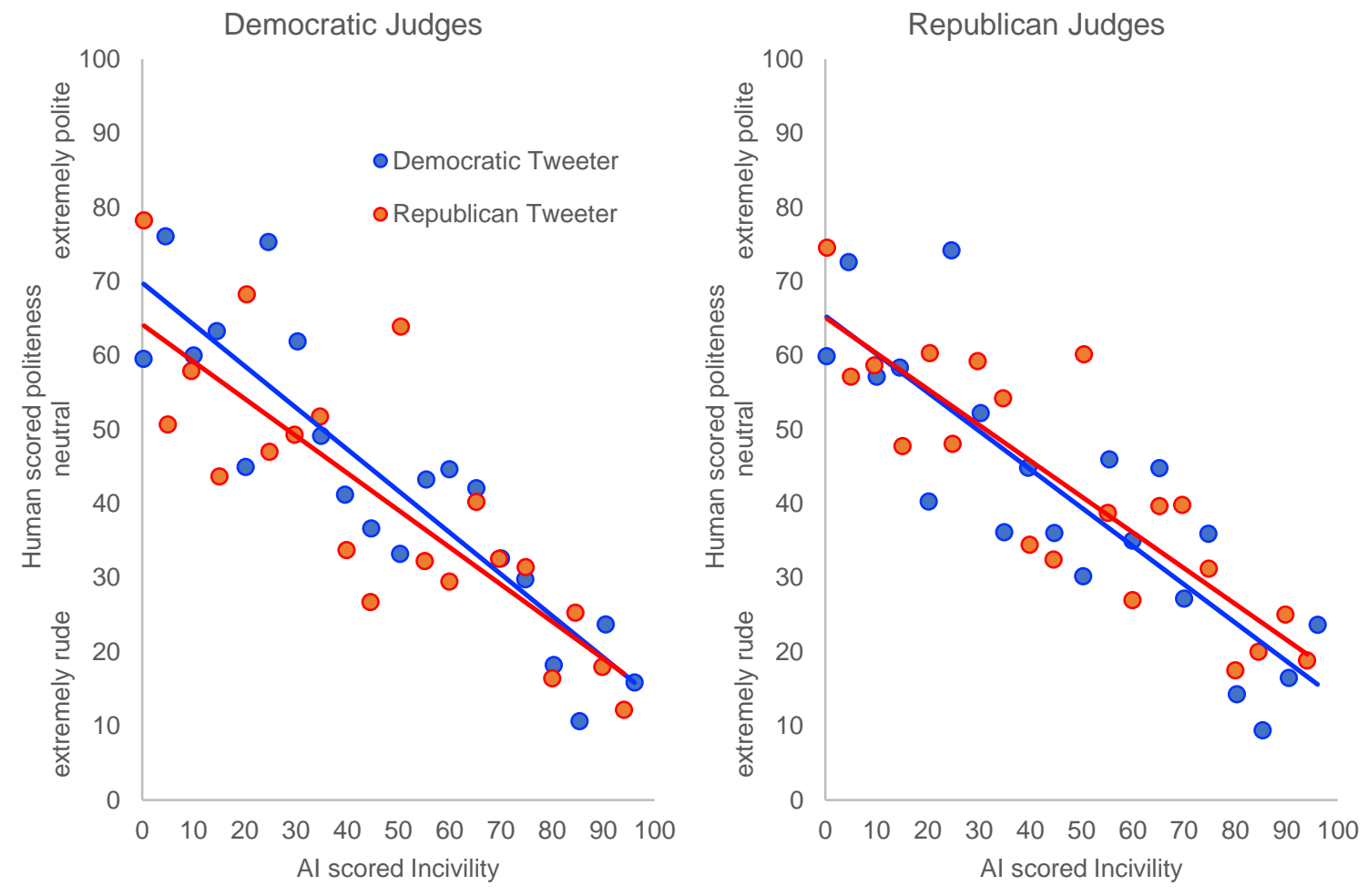
Table S13. Predictors of AI-scored incivility.

\begin{tabular}{lll}
\hline & $\mathrm{B}[95 \% \mathrm{CI}]$ & \\
Predictor of & All Tweets & Incivility $<.40$ \\
\hline Computer-Scored Incivility & $76.613[74.795,78.431]^{* * *}$ & $30.114[28.407,31.821]^{* * *}$ \\
Judged Politeness & $-0.601[-0.629,-0.573]^{* * *}$ & $-0.160[-0.184,-0.135]^{* * *}$ \\
Twitter User's Party & $-0.339[-1.867,1.189]$ & $1.229[-0.479,2.936]$ \\
Judge's Party & $-1.273[-3.181,0.634]$ & $0.114[-1.669,1.897]$ \\
Politeness $\times$ Twitter User & $0.003[-0.024,0.03]$ & $-0.022[-0.047,0.002] \dagger$ \\
Politeness $\times$ Judge & $0.028[-0.001,0.057] \dagger$ & $0.000[-0.025,0.025]$ \\
Twitter User $\times$ Judge & $0.964[-0.636,2.564]$ & $-0.642[-2.426,1.141]$ \\
Politeness $\times$ Twitter User $\times$ Judge & $-0.002[-0.030,0.026]$ & $0.013[-0.013,0.038]$ \\
\hline Note. $\dagger p<.10,{ }^{* * *} p<.001$. & &
\end{tabular}

The marginal politeness $\times$ judge effect suggests that Democrats' politeness judgments tracked $11 \%$ more strongly with AI-scored incivility, $\mathrm{B}=-0.618,95 \% \mathrm{CI}=[-0.659,-0.577], p<.001, \beta$ $=.647$, than did those of Republicans, $\mathrm{B}=-0.555,95 \% \mathrm{CI}=[-0.597,-0.513], p<.001, \beta=.581$. Noting that our sample of tweets spanned the full spectrum of incivility whereas the sample of tweets in Study 1 clustered near the low end of incivility, we re-ran this analysis with only tweets with AI-scored incivility <.40. The pattern of results was unchanged (see Table S11).

Next, we tested whether AI-scored incivility is reducible to negatively stated opinions by adding judgments of tone and opinion judgments as predictors of AI-scored incivility. We found that AI-scored incivility was associated with being seen as opinion-based, $\mathrm{B}=0.146,95 \% \mathrm{CI}=$ $[0.118,0.174], p<.001$, and with a negative tone, $\mathrm{B}=-0.179,95 \% \mathrm{CI}=[-0.217,-0.141], p<$ .001 . However, politeness judgments still predicted AI-coded incivility, $\mathrm{B}=-0.427,95 \% \mathrm{CI}=[-$ $0.464,-0.389], p<.001$, meaning that AI-scored incivility is not reducible to negative opinions. Finally, we examined whether AI-scored incivility's validity generalized to people who are and who are not on Twitter. For this analysis, we included politeness judgments, whether (1) or not (0) judges reported being on Twitter, and their interaction to predict AI-coded incivility. We again found a strong effect of judged politeness, $\mathrm{B}=-0.649,95 \% \mathrm{CI}=[-0.710,-0.587], p<.001$. There was no effect of whether participants were on Twitter, $\mathrm{B}=-2.11,95 \% \mathrm{CI}=[-6.55,2.33], p$ $=.352$, nor was there an interaction, $\mathrm{B}=0.062,95 \% \mathrm{CI}=[-0.007,0.131], p=.076$. However, AIscored incivility was a valid measure of politeness for people on Twitter, $\mathrm{B}=-0.584,95 \% \mathrm{CI}=[-$ $0.615,-0.552], p<.001$, and for people not on Twitter, $\mathrm{B}=-0.659,95 \% \mathrm{CI}=[-0.718,-0.601], p$ $<.001$. These results suggest that PerspectiveAPI is a valid and generalizable tool for measuring incivility in political tweets.

\section{Study S2: Many “Likes” Does Not Imply Approval 1}

Does a tweet receiving many "likes" mean that the average Twitter user who saw a tweet necessarily approved of the tweet? The goal of Study S2 was to test the validity of Twitter metrics as indicators of observers' evaluations in a sample of tweets by members of U.S. Congress over a relatively long period of time (October 2014 - July 2019). We used the sentiments of actual responses/comments to the original tweets on Twitter as a measure of the Twitter community's feelings about the tweet (Study S3 uses explicit off-Twitter judgments). 
We used software to code the emotional tone of the replies to tweets, with the original tweets having been issued by members of Congress. We then tested whether the tone of the comments predicted the three Twitter metrics_-"retweets", "likes", and "likes"-per-"retweet".

\section{Method}

Procedure. We collected 46,234 replies to 3,586 tweets issued by 363 members of Congress, then used Vader sentiment ("compound" variable) to score the emotional tone of each reply on a scale from -1 to 1. Emotional tone scores reflect how negative/ disapproving (lower scores) or positive/approving (higher scores) a statement is.

Analytic Strategy. All three metrics ("retweets", "likes", and "likes"-per-"retweet") evidenced skewed distributions (see Table S14) so we performed a log transformation before standardizing the metrics (z-scores). This standardization had the effect of making multilevel model estimates tantamount to standardized effect sizes ( $\beta \mathrm{s})$ and thus comparable between analyses and studies. We estimated effects within a 3-level multilevel model with random intercepts for each tweet and each member of Congress.

Table S14. Descriptive statistics of Twitter metrics and reply sentiments in the samples of tweets by members of U.S. Congress (Study S2).

\begin{tabular}{|c|c|c|c|c|c|c|}
\hline \multirow[b]{2}{*}{ Metric } & \multicolumn{3}{|c|}{ Raw } & \multicolumn{3}{|c|}{ Log Transformed } \\
\hline & $M(S D)$ & Range & Skew & $M(S D)$ & Range & Skew \\
\hline Sentiment & $-0.05(0.49)$ & -0.99 to 0.98 & 0.01 & & & \\
\hline "Retweets" & $1,705.13(5,862.12)$ & 0 to 83,609 & 6.31 & $1.83(1.17)$ & -1.00 to 4.92 & 0.34 \\
\hline "Likes" & $6,300.62(21,001.92)$ & 0 to 259,577 & 5.18 & $2.37(1.15)$ & -1.00 to 5.41 & 0.47 \\
\hline "Likes"-per-"Retweet" & $4.87(11.93)$ & 0.05 to 870 & 31.44 & $0.54(0.29)$ & -1.30 to 2.94 & 0.85 \\
\hline
\end{tabular}

\section{Results}

To observe the trends, we plotted the three metrics against reply sentiments in Figure S4. These figures show how the tweets that elicited the most positive comments tended to receive relatively few "likes." They also received few "retweets". In contrast, they received many "likes" per "retweet". Formally, the greater number of replies a tweet received, the lower the sentiment of replies to the tweet, $\beta=-.090,95 \% \mathrm{CI}=[-.115,-.065], p<.001$. And as "likes" increased, sentiment became more negative, $\beta=-.079,95 \% \mathrm{CI}=[-.105,-.053], p<.001$. In contrast, the "likes"-per-"retweet" ratio correlated positively with sentiments, $\beta=.034,95 \% \mathrm{CI}=[.017, .052]$, $p<.001$. These results suggest that the "likes" metric may not be a valid measure of positive sentiment and that a "likes"-per-"retweet" ratio might succeed in revealing the sentiment of the Twitter community where the "likes" metric fails. An alternative interpretation is that people on Twitter use the "likes" metric to express approval and the "reply" option to express disapproval, and uncivil tweets tend to elicit more of both than civil ones. We therefore elicited explicit social evaluations in Study S3. 
Figure S4. Average sentiments of replies to tweets that varied in their level of incivility.
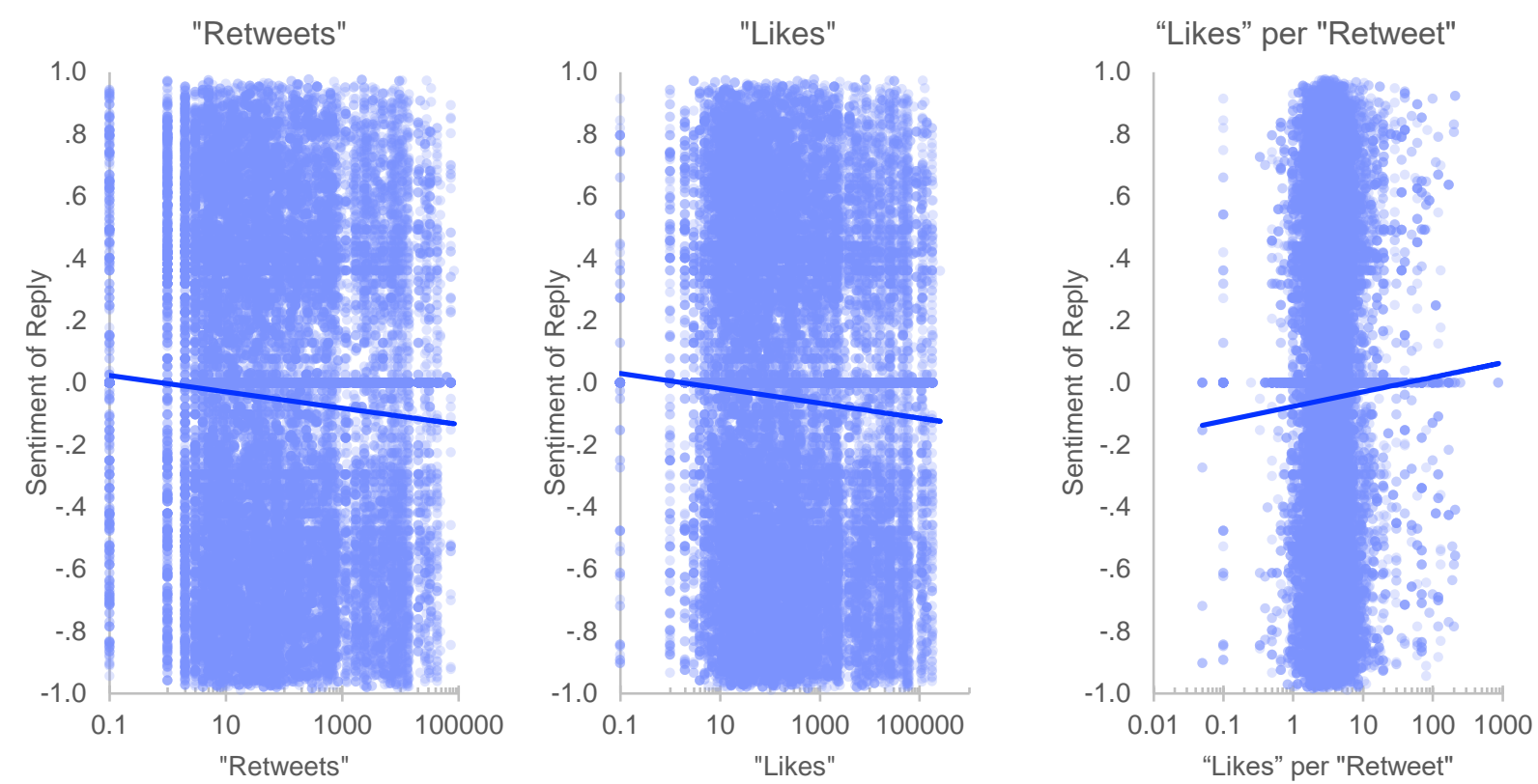

\section{Study S3: Many “Likes" Does Not Imply Approval 2}

The goal of Study S3 was to test whether people explicitly approve or disapprove of tweets that happen to have received many "likes" using a sample of tweets by members of U.S. Congress. We recruited a politically diverse panel of Americans off of Twitter to read and report their level of approval toward 40 tweets by members of the U.S. Congress without knowing how many "likes" or "retweets" it had received. We then tested whether their approval ratings correlated with the two Twitter metrics ("likes", "retweets") and a "likes"-per-"retweet" ratio.

\section{Method}

Judges. The judges were 157 Americans recruited on Amazon's TurkPrime. Because most Mechanical Turk samples are predominantly liberal, we used responses to pre-screening questions to recruit an ideologically diverse panel, with 56 liberals, 53 moderates, and 45 conservatives. Most participants $(71 \%)$ reported that they were on Twitter. The sample was $64 \%$ male, $76 \%$ White, and 37 years old on average $(S D=11)$. As each of the 157 observers rated all 40 tweets, there were 6280 judgments in total.

Procedure. Each judge reviewed 40 tweets with each tweet having been issued by a unique sitting member of U.S. Congress; observers then indicated their level of approval toward each tweet. They then completed a self-affirmation task to mitigate potential negative affect and reported demographics.

Tweets. There were 535 members of the $116^{\text {th }}$ Congress of the United States. To build a corpus of stimuli (tweets), we randomly sampled 40 members of Congress and then randomly sampled one tweet from each of the sampled members issued between May 1 - May 31, 2019. For each tweet, we presented an image of the original tweet with the metrics ("likes", "retweets") hidden from view. We opted to include 40 tweets to strike a balance between making the corpus representative (the more tweets the better for this purpose) while avoiding overtaxing observers who were to read and respond to all of them (fewer tweets is better for this purpose). Tweets were presented in random order for each participant. For each tweet, we gathered metrics 
regarding the number of times the tweet had been "liked" and "retweeted" on Twitter, and then computed a "likes"-per-"retweet" ratio.

Approval. The question asked, "How do you feel about this tweet?" Judges responded on an 11point scale anchored at -5 (extremely negative), -3 (negative), -1 (slightly negative), 0 (neutral), 1 (slightly positive), 3 (positive), 5 (extremely positive).

Demographics. Along with reporting gender, age, and race, participants reported their political ideology, as liberal (scored -1), moderate (0), or conservative (1). They also indicated whether they were on Twitter (scored 1) or not (0).

Analytic Strategy. All three Twitter metrics had skewed distributions so we log-transformed each one to produce normal distributions (see Table S15). We then computed z-scores for each metric and for judge's sentiments. This standardization had the effect of making multilevel model estimates equivalent to standardized effect sizes ( $\beta \mathrm{s})$ and thus comparable between analyses and studies.

Table S15. Skewness statistics of Twitter metrics and judge's sentiments in the samples of tweets by members of U.S. Congress (Study S3).

\begin{tabular}{lllllll} 
& & \multicolumn{3}{c}{ Raw } & \multicolumn{3}{c}{ Log Transformed } \\
Metric & $\boldsymbol{M}(S D)$ & \multicolumn{1}{c}{ Range } & Skew & $\boldsymbol{M}(\boldsymbol{S} D)$ & Range & Skew \\
\hline Approval & $1.29(2.89)$ & -5 to 5 & -0.48 & & & \\
"Likes" & $850(1399)$ & 20 to 6000 & 2.40 & $2.50(0.60)$ & 1.30 to 3.78 & 0.47 \\
"Retweets" & $247(418)$ & 8 to 2200 & 3.13 & $1.98(0.59)$ & 0.90 to 3.34 & 0.53 \\
"Likes" per "Retweet" & $3.89(2.65)$ & 1.22 to 16.65 & 3.12 & $0.53(0.23)$ & 0.08 to 1.22 & 0.61 \\
\hline
\end{tabular}

\section{Results}

We estimated the association between each metric and judges' approval using multilevel models. We included random intercepts for each judge. The basic model (see Table S14) included only Twitter metrics as predictors of approval in three separate models, one for each metric. Like in Study S2, we found that the "retweets" and "likes" metrics correlated negatively with approval. This means that disliked tweets were "retweeted" and "liked" more than liked tweets.

Counterintuitively, the more "likes" a tweet received, the more negative research participants reported feeling about the tweet. To illustrate these results, we plotted each metric against the average approval across all judges in Figure S5. Unlike the visible metrics, the "likes"-per"retweet" metric correlated positively with judges" approval, meaning that the "likes"-per"retweet" ratio appears to be a valid metric of observer approval. A "likes"-per-"retweet" ratio of 10 was associated with approval whereas a ratio of 1 was associated with neither approval nor disapproval. 
Figure S5. Scatterplots of the average approval (across all judges) and the three Twitter metrics (Study S3). Each dot represents a tweet by a member of U.S. Congress. Metrics were log transformed before analyses to reduce skewness.
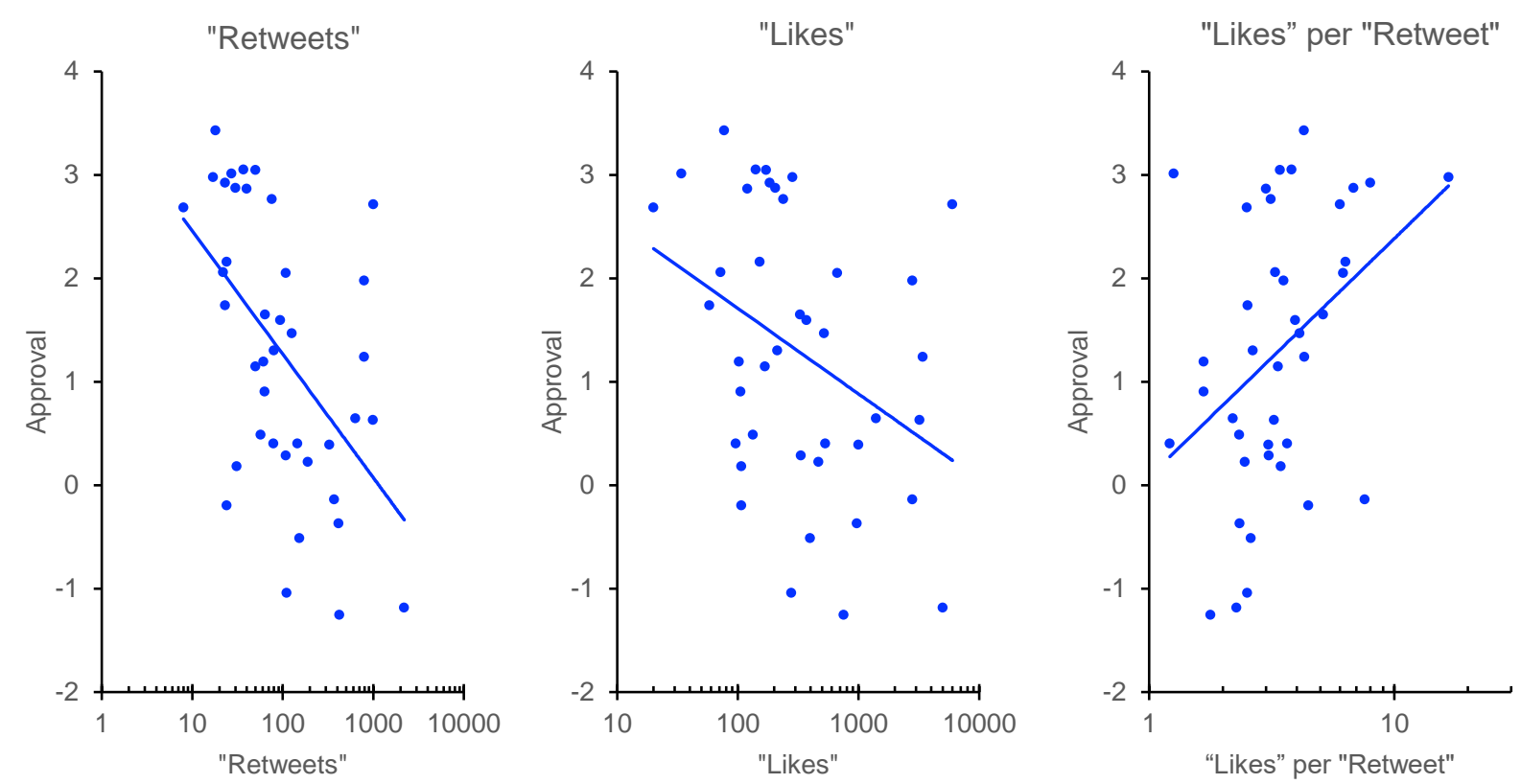

Did the ideology of the judge and/or the party of the tweeter condition how the metrics track approval? We thus tested the generalizability of the validity of metrics as indicators of approval by examining whether the positive association between the metric and approval was limited to judges of a particular political ideology, observers who are or are not on Twitter, and to tweets by Democratic or Republican politicians. To this end, we added all three main effects and all possible interactions to the base model to create full factorial models (see Table S16). These models showed that the "likes"-per-"retweet" metric appears to be a valid indicator of approval of observers from across the ideological spectrum. The main effect of "likes"-per-"retweet" reproduced the evidence of the validity of "likes"-per-"retweet" as a metric of approval and the "likes"-per-"retweet" did not significantly interact with any of the three contextual factors, nor with their higher order interactions. That is, we found evidence that the validity of "likes"-per"retweet" as a metric of approval generalizes across the political spectrum and for people who are or are not on Twitter. To illustrate this invariance, we plotted the approval and "likes"-per"retweet" metric for liberals, moderates, and conservatives separately in Figure S6. 
Table S16. Predictors of the approval of an ideologically balanced panel of judges toward 40 tweets by members of the U.S. Congress. All metrics ("likes"-per-"retweet", "likes", and "retweets") were log transformed and then standardized. Approval was also standardized. Analyses were multilevel models with random intercepts for each judge. Numbers are unstandardized estimates but because of the standardization of all variables before analyses, all numbers in effect represent standardized estimates $(\beta \mathrm{s})$.

\begin{tabular}{|c|c|c|c|c|c|c|}
\hline \multirow[t]{3}{*}{ Predictors of Approval } & \multicolumn{6}{|c|}{ Metric (Log Transformed and then Standardized) } \\
\hline & \multicolumn{2}{|c|}{ “Likes" per "Retweet" } & \multicolumn{2}{|c|}{ "Likes" } & \multicolumn{2}{|c|}{ "Retweets" } \\
\hline & Basic Model & Full Factorial & Basic Model & Full Factorial & Basic Model & Full Factorial \\
\hline tric & $.178[.154, .201]^{* * *}$ & $.130[.088, .172]^{* * *}$ & $-.240[-.262,-.217]^{* * *}$ & $-.200[-.240,-.159]^{* * *}$ & $-.169[-.192,-.146]^{* * *}$ & $-.255[-.324,-.186]^{* * *}$ \\
\hline Ige's Conservatism & & $-.096[-.217, .025]$ & & $-.101[-.221, .02]$ & & $.248[-.007, .502] \dagger$ \\
\hline Twitter & & $.107[-.005, .218] \dagger$ & & $.103[-.008, .214] \dagger$ & & $.181[-.054, .416]$ \\
\hline reeter Party & & $.167[.125, .209]^{* * *}$ & & $.184[.144, .223]^{* * *}$ & & $-.191[-.368,-.013]^{*}$ \\
\hline tric $\mathrm{x}$ Conservatism & & $.012[-.042, .067]$ & & $-.081[-.133,-.028]^{* *}$ & & $-.142[-.232,-.053]^{* *}$ \\
\hline tric $\mathrm{x}$ Twitter & & $.039[-.011, .089]$ & & $-.034[-.082, .015]$ & & $-.030[-.112, .053]$ \\
\hline tric x Party & & $-.009[-.051, .033]$ & & $.076[.035, .116]^{* * *}$ & & $.156[.087, .225]^{* * *}$ \\
\hline nservatism x Twitter & & $.026[-.117, .169]$ & & $.033[-.109, .175]$ & & $-.223[-.523, .077]$ \\
\hline nservatism x Party & & $.384[.330, .439]^{* * *}$ & & $.382[.330, .433]^{* * *}$ & & $-.005[-.236, .225]$ \\
\hline itter x Party & & $-.083[-.133,-.033]^{* *}$ & & $-.077[-.125,-.029]^{* *}$ & & $.067[-.146, .279]$ \\
\hline tric $\mathrm{x}$ Conservatism $\mathrm{x}$ Twitter & & $-.035[-.099, .029]$ & & $.071[.009, .133]^{*}$ & & $.103[-.003, .208] \dagger$ \\
\hline tric x Conservatism x Party & & $-.051[-.106, .003] \dagger$ & & $.11[.057, .162]^{* * *}$ & & $.157[.068, .246]^{* *}$ \\
\hline tric $x$ Twitter x Party & & $-.009[-.059, .041]$ & & $-.036[-.084, .012]$ & & $-.056[-.139, .026]$ \\
\hline $\begin{array}{l}\text { nservatism } x \text { Twitter } x \text { Party } \\
\text { tric } x \text { Twitter } x \text { Party } x\end{array}$ & & $-.107[-.171,-.043]^{* *}$ & & $-.110[-.171,-.049]^{* * *}$ & & $-.069[-.341, .202]$ \\
\hline nservatism & & $.037[-.027, .101]$ & & $-.019[-.081, .043]$ & & $-.018[-.124, .087]$ \\
\hline
\end{tabular}


Figure S6. Average approval of liberals, moderates, and conservatives toward 40 tweets my members of the U.S. Congress and their respective "likes"-per-"retweet" ratios.
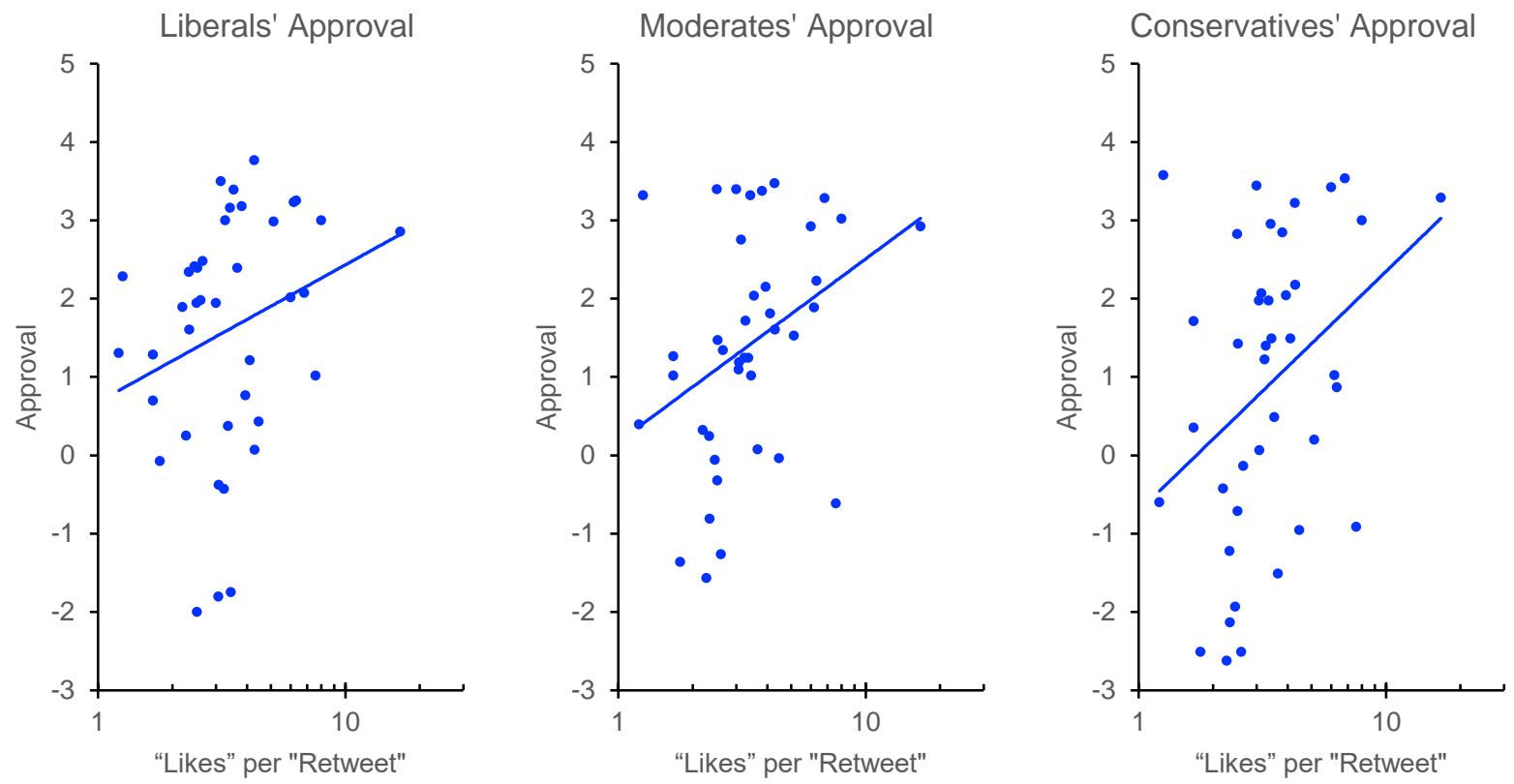

Tangential to the question of validity, we found that tweets by Republican members of Congress were more "liked" than those issued by Democratic members (main effect of Party). We also found an ingroup bias (ideology $\times$ party interaction). For Democratic politicians' tweets, conservativism negatively predicted approval $(\beta=-.400, p<.001)$ whereas conservatism positively predicted approval towards tweets by Republican members of Congress, $(\beta=.220, p<$ .001). This ingroup preference was further qualified by whether or not the judge was on Twitter (ideology $\times$ on Twitter $\times$ party interaction). Respondents who were not on Twitter showed stronger ingroup biases $\left(\beta_{\text {Republican Politicians }}=-.493\right.$ and $\left.\beta_{\text {Democratic Politicians }}=.280, p \mathrm{~s}<.001\right)$ than did people on Twitter $\left(\beta_{\text {Republican Politicians }}=-.345\right.$ and $\left.\beta_{\text {Democratic Politicians }}=.198, p \mathrm{~s}<.001\right)$. Finally, we found an on-Twitter $\times$ Party interaction such that people who were not on Twitter showed a stronger preference for Republican over Democratic tweets $(\beta=.228, p<.001)$ than people who were on Twitter $(\beta=.083, p<.001)$.

Our efforts to derive a metric that tracks with approval led to a correction for overall exposure of the tweet, which prompted a division by the number of "retweets" a tweet received. However, "retweets" can cause exposure to grow exponentially, as "retweeting" can lead to more exposure, which can lead to more "retweeting" and exposure. Thus, dividing by "retweets" to the first power may not be ideal and the "retweets" metric should be raised to some power greater than 1 . To test this idea, we empirically modeled the approval as being related to "likes" per "retweets" to a variable exponent:

$$
\text { Approval }=a+b \frac{\text { "Likes" }}{\text { "Retweets"c }}+\varepsilon
$$

Both "likes" and "retweets" were log-transformed in this analysis. The constant, $c$, was assumed to be 1 in the prior analyses but is a fit parameter in this one. When $c=1$, the model fits the data at $R^{2}=.153$. A least sum-of-squares solution to (1) yielded an exponent $c=1.508$ and fit the data 
at $R^{2}=.344$. This result suggests that an optimal approval metric might be non-linearly related to the number of "retweets".

\section{Discussion}

This study found that neither "likes" nor "retweets" predict overt approval. In fact, both metrics correlated negatively with approval. This is particularly surprising for the "likes" metric and supports the seemingly self-contradictory statement that tweets that received many "likes" were probably poorly received by observers. This paradox is likely explained by unfavorably received tweets getting more attention and exposure than favorably received ones.

The "likes"-per-"retweet" was again a valid indicator of approval in a sample of tweets by various politicians and with explicit judgments of approval. The validity of this metric generalized to predicting the approval of liberals, moderates, and conservatives, meaning that the "likes"-per-"retweet" ratio is likely a valid indicator of the approval of observers from across the political spectrum. This is particularly important because the ideological leaning of the people that "retweet" and "like" tweets in large corpuses such as our study of the U.S. Congress is often unknown. The current results suggest that this blind spot might not be consequential with respect to drawing inferences from the "likes"-per-"retweet" metric about the approval of Twitter users. Effect sizes indicative of validity were relatively small, meaning that "likes"-per-"retweet" is not a perfect indicator of approval, and that there is room for developing an even more valid metric. However, in large data sets, a small signal may be sufficient to pick up important trends. In contrast,

\section{Study S4: Many "Retweets" Does Not Imply Approval}

Why do people "retweet" uncivil tweets? Past research (Metaxas et al., 2017) found that people retweet as a way of expressing various forms of positivity toward a tweet. However, this past study focused on retweeting in general, and did not explore why people might retweet uncivil messages. In the present study, we examined three possibilities using a mediation approach. First, people might "retweet" uncivil tweets because they find them to be more entertaining than civil tweets and want to share something interesting with their followers. Second, people might "retweet" uncivil tweets because they morally approve of the verbal attack on a mutually disliked target. Alternatively, people might "retweet" uncivil tweets because they dislike them and want to "retweet" (perhaps with a disapproving comment) to call out the norm violation and/or express their disapproval of verbal attacks. And third, people might anticipate that their friends and followers will feel the same way as they do about uncivil tweets, and "retweeting" will thus start or add to a bandwagon effect.

\section{Method}

Participants. The sample was 161 Americans on TurkPrime; it was $60 \%$ male, $74 \%$ white, and $78 \%$ of participants reported that they were on Twitter. The average participant was 37 years old $(S D=12)$. We again used pre-screening questions to achieve a politically balanced sample. Politically, the sample was 36\% Republican, 43\% Democrat, and 22\% Independent. Each participant received $\$ 1.00$.

Procedure. Participants reviewed 20 tweets and rated how uncivil and entertaining each tweet was, how much they disliked it, and the degree to which their friends would share their view about the tweet. They then answered two questions about their social motives, reported demographics (for an unrelated purpose; not analyzed here), and were debriefed. 
Tweets. The sample of tweets was from sitting Democrats and Republicans in Congress that represented the full spectrum of incivility (see Study S3 for the list). We coded the level of incivility of each tweet using PerspectiveAPI then randomly selected original tweets that had a AI-scored incivility score of $0,10,20, \ldots, 80,90$. Thus, there were 10 tweets by Democrats and 10 tweets by Republicans. We removed URLs and emojis. Participants read all 20 tweets in random order. They saw only the text of the tweet. They were not made aware of the name or party of the tweeter or provided any context. The tweets are shown in Table S10. We used the actual number of times that each tweet was "retweeted" on Twitter as our measure of "retweets" (log-transformed).

Judgments. Participants judged each tweet in terms of four qualities. First, they rated how uncivil each tweet was. Below the tweet text, a question asked, "How uncivil is the tweet?" Reponses were on a 7-point scale anchored at -1 (very civil), -0.67 (somewhat civil), -0.33 (slightly civil), 0 (neutral), 0.33 (slightly uncivil), 0.67 (somewhat uncivil), and 1.00 (extremely uncivil). The second question asked, "How entertaining is the tweet?" Responses were on a similar 7-point scale with the word civil replaced with dull and uncivil replaced with entertaining. And the third question assessed sentiments, "How do you feel about the tweet?" Responses were on a similar scale, ranging from very negative to very positive. And the fourth question assessed the bandwagon effect, the degree to which participants thought their friends would share their feelings about the tweet: "How do you think most of your friends would feel about the tweet?", with response options ranging from very different from me to very similar to me.

Demographics. Along with age, gender, and race, participants reported their party identification (Democrat, Independent, Republican), ideology (extremely liberal to extremely conservative on a 7-point scale), and whether they are on Twitter.

\section{Results}

Each participant made 4 judgments on each of the 20 tweets. We used multilevel models to estimate relationships, which included random intercepts for each judge. First, we tested whether tweets that were judged to be uncivil were more entertaining, disliked, and likely to generate a bandwagon effect in three separate multilevel models (each had one incivility as the predictor and one mediator as the outcome). We found that uncivil tweets were more entertaining and disliked, but less likely to support a bandwagon effect (as in, they were divisive; see Figure S7). Second, we tested the partial relationships between the mediators and the number of "retweets" by entering incivility and the three mediators as simultaneous predictors of the number of "retweets" in a multilevel model. Here, we found that tweets that were found to be entertaining received more "retweets", but disliked tweets and bandwagon supporting tweets did not. And third, we found that the tendency for uncivil tweets to receive more "retweets" was somewhat diminished when controlling for these three mediating factors.

Figure S7. Results of mediation analyses explaining why people tend to "retweet" uncivil tweets. The three tested explanations were that people "retweet" uncivil tweets because they find them entertaining (supported), because they dislike them and want to express their disapproval (unsupported), and to create or join the bandwagon (they think their peers agree with them about the uncivil tweet; unsupported). Statistics represent unstandardized estimates from multilevel models. Solid lines and boldface font represent statistically significant results. Dotted lines and regular font represent effects that did not reach significance. ${ }^{* * *} p<.001$ 


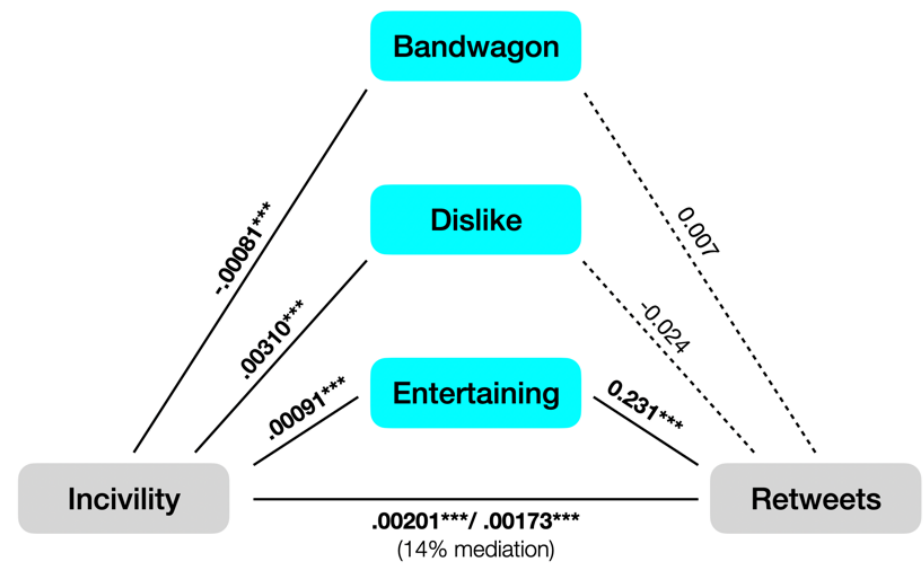

\section{Discussion}

Study S4 found that uncivil tweets were "retweeted" because they were found to be entertaining and/or funny. Whereas past research (Metaxas et al., 2017) found that people "retweet" in general to express approval, we found no evidence that the elevated retweeting of uncivil tweets per se was linked to approval. Thus, the elevated "retweeting" of uncivil tweets is not an indication that the Twitter community approved of the content.

\section{Study S5: Congressional Speeches \& Presidential Debates}

To explore whether the rise in incivility on Twitter generalized to other contexts, we examined trends in incivility in congressional speeches and presidential debates over decades.

\section{Methods}

Congressional speeches. We downloaded the U.S. Congressional Record, which contains the verbatim transcripts of all speeches delivered on the floor of the U.S. Congress between January 4, 1995 (when transcription began) and January 31, 2020 from https://github.com/billegar1/congress.gov-scrape. The data set had 432,312 speeches in total, which contained 5,046,910,384 words. Names of the speakers were available for fewer than $40 \%$ of speeches so we did not attempt to unite the Twitter and Congressional Record data sets. The number of speeches in Congress was relatively uniform over the period of study.

Presidential debates. We acquired the transcripts of the Presidential debates between 1976-2016 from https://www.debates.org/voter-education/debate-transcripts/, which spanned the entire time span of debate transcripts without gap election cycles (the database has the 1960 transcript, then skips 1964-1972). Each debate transcript divided the text into statements (paragraphs). We therefore used the statement as the unit of analysis. There were 13,213 statements across all years, 4,089 of which were not from a Democrat or Republican Presidential or Vice-Presidential candidate (e.g., moderators, audience members). We excluded these in all analyses, leaving 9,124 statements by Democratic and Republican nominees. Statements were 53 words long on average $(S D=68)$, range $1-765$.

Incivility. To code the incivility of each statement, we had Python code transmit one speech/debate statement per second to http://perspectiveapi.com. PerspectiveAPI can analyze any text up to 3000 characters in length. No debate statements exceeded the 3000-character limit. 
However, $9.2 \%$ of Congressional speeches were longer than 3000 characters. For these long speeches, we split the text into 3000-character segments, analyzed the incivility of each, and then averaged the incivility across all segments for each speech. A few $(0.4 \%)$ of speeches were more than 60,000 characters in length; for these, we analyzed and averaged the first 60,000 characters (20 segments).

\section{Results}

Congressional speeches. We tested whether incivility increased or decreased over time in speeches of members of U.S. Congress and in Presidential debates (Figure S8). A multilevel model with random intercepts for each chamber of government (Senate vs. House of Representative) revealed that incivility declined over the 25 years under study from 12.2 in 2009 to 9.9 in 2019 , representing a $19 \%$ decrease from. Incivility declined precipitously between 2010-2011, which was around the time that Twitter began to be widely used. Was the overall decline in incivility attributable to the precipitous decline in early 2011 ? To test this possibility, we split the sample on January 1, 2011, and tested whether incivility declined before and after that date. Our analyses confirmed that incivility declined in the Congressional Record both before and after 2011 (see Table S17).

Figure S8. Changes in levels of incivility in Congressional speeches and presidential debates. Error bars represent $95 \%$ CIs.
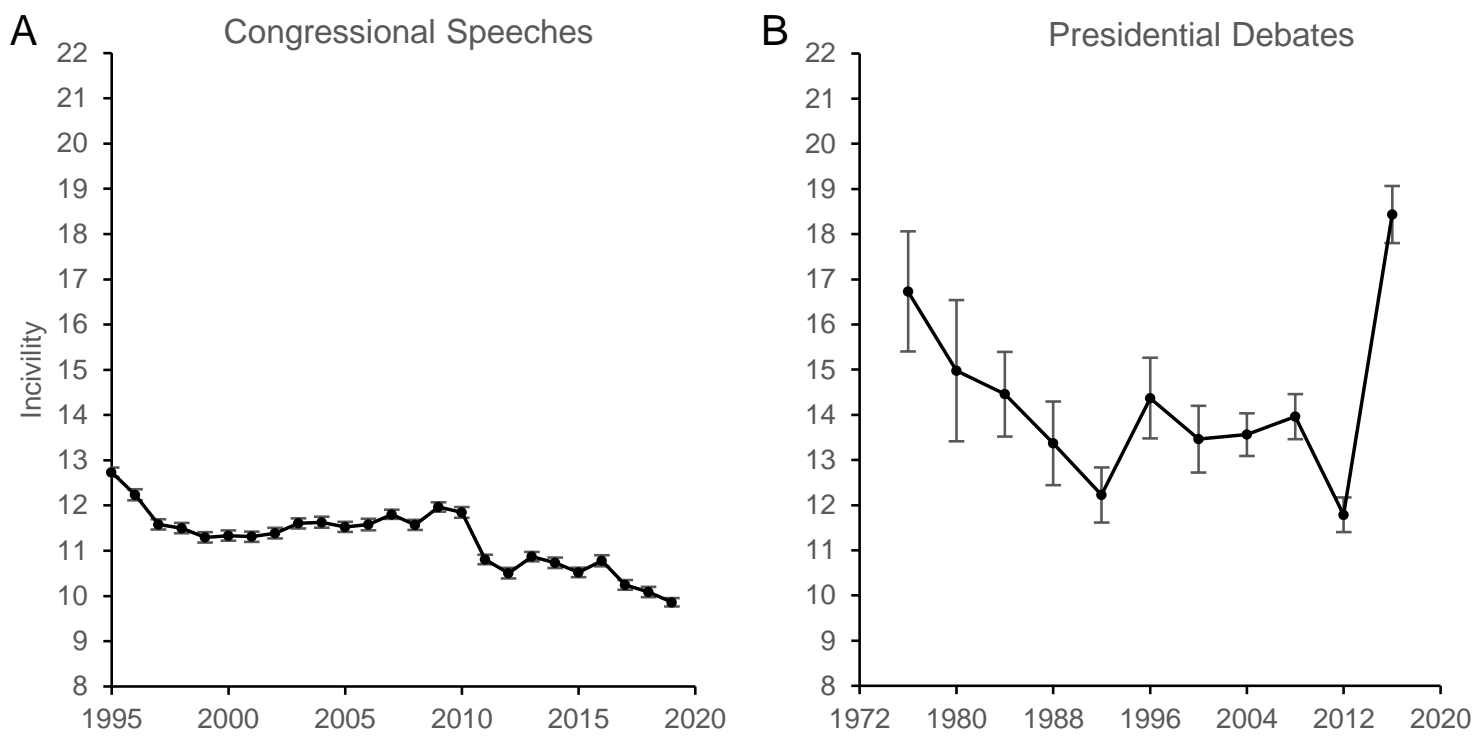

Table S17. Results from multilevel models testing whether incivility increased or decreased over time in the floor speeches of members of Congress (in the Congressional Record) in the spans of 1995-2020, 1995-2010, and 2011-2020. Time was in units of years, and centered at the mean (mid-2007). Numbers in brackets represent $95 \%$ confidence intervals. 回 $0<.10,{ }^{* *} p<.01,{ }^{* * *} p<.001$.

\begin{tabular}{llll}
\hline & & \multicolumn{2}{c}{ Time Span } \\
Predictor of Incivility & $\mathbf{1 9 9 5 - 2 0 2 0}$ & $\mathbf{1 9 9 5 - 2 0 1 0}$ & $\mathbf{2 0 1 1 - 2 0 2 0}$ \\
\hline Intercept & $11.21[5.68,16.74]^{* * *}$ & $11.59[-0.08,23.27]$ ?] & $11.29[6.47,16.12]^{*}$ \\
Time (years) & $-0.085[-0.088,-0.082]^{* * *}$ & $-0.023[-0.029,-0.017]^{* * *}$ & $-0.089[-0.102,-0.075]^{* * *}$ \\
\hline
\end{tabular}

Presidential debates. We conducted a multilevel model, with random intercepts for each debate. Predictors of incivility were time (in years), political party ( 1 = Republican, -1 = Democrat), and 
their interaction. Including all years (1976-2016), we found no effect of time, a main effect of party (Democrats scored higher on incivility), and an interaction.

Observing Figure S8, we can see that the 2016 debates deviated from the historical trend. We observed in the analysis of Presidential tweets that President Trump's language tended to be more uncivil than his counterparts, and reactions to President Trump tended to be more uncivil than other statements from the same politicians. We therefore considered trends in incivility prior to the 2016 debates (see Table S18) and found that incivility declined in Presidential debates prior to 2016, a result that mirrors the general decline in incivility in the speeches of Congress between 1995-2019.

Table S18. Results from multilevel models testing whether incivility increased or decreased over time in Presidential debates, and whether it depended on the political party ( $1=$ Republican, -1=Democrat) in 44 years of Presidential debates (1976-2016) and in the pre-Trump era (1976-2012). Time was in units of years. Numbers in brackets represent $95 \%$ confidence intervals. $0 ?$ l $p<.10,{ }^{*} p<.05,{ }^{* *} p<.01$.

Predictor of Incivility

Time Span

1976-2016

1976-2012

\begin{tabular}{lll}
$\begin{array}{l}\text { All Presidential Candidates } \\
\text { (Intercept) }\end{array}$ & $18.129[-125.605,161.863]$ & $192.905[72.645,313.165]^{* *}$ \\
Time & $-0.002[-0.074,0.070]$ & $-0.090[-0.150,-0.030]^{* *}$ \\
Party & $-47.889[-90.135,-5.644]^{*}$ & $-38.013[-83.799,7.774]$ \\
Time $\times$ Party & $0.024[0.003,0.045]^{*}$ & $0.019[-0.004,0.042]$ \\
Democrats & & \\
Time & $-0.032[-0.11,0.047]$ & $-0.106[-0.175,-0.038]^{* *}$ \\
Republicans & & \\
Time & $0.028[-0.059,0.115]$ & $-0.068[-0.137,0.000] \dagger$ \\
\hline
\end{tabular}

\section{References}

Boxell, L., Gentzkow, M., \& Shapiro, J. M. (2017). Greater Internet use is not associated with faster growth in political polarization among US demographic groups, PNAS 114(40), 10612-10617. Doi: 10.1073/pnas.1706588114

Brady, W. J., Wills, J. A., Burkart, D., Jost, J. T., \& Van Bavel, J. J. (2019). An ideological asymmetry in the diffusion of moralized content on social media among political leaders. Journal of Experimental Psychology: General, 148(10), 18021813. https://doi.org/10.1037/xge0000532

Lai, N. (2018). Blood on the floor: How did politics become so uncivil. Retrieved from https://www.thebigq.org/2018/09/19/blood-on-the-floor-how-did-politics-become-souncivil/

Mason, L. (2018, June 2018). Here's how political science explains the GOP's obsession with civility. Retrieved from https://www.washingtonpost.com/news/posteverything/wp/2018/06/28/heres-howpolitical-science-explains-the-gops-obsession-with-civility/ 
Metaxas, P. \& TwitterTrails Research Team (2017). Retweets indicate agreement, endorsement, trust: A meta-analysis of published Twitter research. Working paper retrieved from http://cs.wellesley.edu/ pmetaxas/WorkingPapers/Retweet-meaning.pdf

Sap, M., Card, D., Gabriel, S., Yejin, C. \& Smith, N. (2019). The risk of racial bias in hate speech detection. Proceedings of the 57th Annual Meeting of the Association for Computational Linguistics, 1668-1678. doi: 10.18653/v1/P19-1163. 